\title{
Targeted desialylation overcomes glyco-immune checkpoints and potentiates the anticancer immune response in vivo
}

Melissa A. Gray ${ }^{1}$, Michal A. Stanczak ${ }^{2,3}$, Han Xiao ${ }^{1}$, Johan F. A. Pijnenborg ${ }^{1}$, Natália R. Mantuano ${ }^{2,3}$, Stacy A. Malaker ${ }^{1}$, Payton A. Weidenbacher ${ }^{1}$, Caitlyn L. Miller ${ }^{4}$, Julia T. Tanzo $^{1}$, Green Ahn ${ }^{1}$, Elliot C. Woods ${ }^{1}$, Heinz Läubli ${ }^{2,3}$, Carolyn R. Bertozzi ${ }^{1,5 *}$

${ }^{1}$ Department of Chemistry, Stanford University, Stanford, California, 94305, USA.

${ }^{2}$ Cancer Immunology Laboratory, Department of Biomedicine, University Hospital, Basel, Switzerland.

${ }^{3}$ Division of Oncology, Department of Internal Medicine, University Hospital, Basel, Switzerland.

${ }^{4}$ Department of Bioengineering, Stanford University, Stanford, California, 94305, USA.

${ }^{5}$ Howard Hughes Medical Institute, Stanford University, Stanford, California, 94305 USA.

${ }^{*}$ Correspondence to: C.R.B. (bertozzi@stanford.edu).

\section{Abstract:}

Currently approved immune checkpoint inhibitor $(\mathrm{ICl})$ therapies targeting the PD-1 and CTLA-4 receptor pathways are powerful treatment options for certain cancers; however, the majority of patients across cancer types still fail to respond. Addressing alternative pathways that mediate immune suppression could enhance $\mathrm{ICl}$ efficacy. One such mechanism is an upregulation of sialoglycans in malignancy, which has been recently shown to inhibit immune cell activation through multiple mechanisms including Siglec receptor binding, and therefore represents a targetable glyco-immune checkpoint. Here, we report the design of a trastuzumab-sialidase conjugate that potently and selectively strips diverse sialoglycans from breast cancer cells in vivo. In a syngeneic orthotopic HER2 ${ }^{+}$breast cancer model, targeted desialylation delayed tumor growth and enhanced immune cell infiltration and activation, leading to prolonged survival of mice with trastuzumab-resistant breast cancer. Thus, antibody-sialidase conjugates represent a promising modality for cancer immune therapy. 


\section{Introduction}

Immune checkpoint inhibitor $(\mathrm{ICl})$ therapies have revolutionized treatment of certain cancers. For example, blocking antibodies against PD-1, PD-L1, and CTLA-4, have eradicated metastatic tumors in some patients, leading to long-term survival ${ }^{1,2}$. Although immune activation can be lifesaving, most patients do not respond or relapse after an initial response, and the underlying mechanisms of primary and secondary resistance are not well understood ${ }^{3}$. Additional immune modulators might be at play, including alternative $T$ cell checkpoints (e.g., TIM-3, LAG-3, and $A 2 A R^{4-6}$ ), innate immune receptors and ligands (e.g., CD47 and $\operatorname{SIRP} \alpha^{7,8}$ ), and enzymes (e.g., IDO and $\left.A D A R 1^{9,10}\right)$. Several of these targets are under clinical evaluation, often in combination with PD-1/PD-L1 blockade ${ }^{11}$.

While most ICls target protein checkpoints ${ }^{11}$, cell-surface glycosylation has recently garnered interest as a mediator of immune inhibition ${ }^{12}$. This is supported by several decades of literature, which have identified altered glycosylation as a hallmark of malignancy ${ }^{13,14}$. One example of a glycosylation pattern associated with cancer transformation is an increase in sialic-acid containing proteins and lipids (sialoglycans), a phenotype that intensifies with tumor progression and enhances tumor growth only in the context of an intact immune system in mice ${ }^{15-17}$. Subsequent work has demonstrated that sialoglycans suppress immune activation and act as glyco-immune checkpoints through multiple mechanisms: blocking complement-dependent cytotoxicity (CDC), inhibiting immune-mediated apoptosis, masking immune-activating ligands, and directly binding the sialic acid-binding immunoglobulin-like lectin (Siglec) receptors ${ }^{15,18-}$ 20. In particular, the Siglec-sialoglycan axis of immune modulation is emerging as an important mediator of sialic acid-induced immune suppression in the context of cancer $^{21}$.

The Siglec receptor family binds to a variety of sialoglycan structures and populates, often in combination, every immune cell class ${ }^{22,23}$. Eight family members (Siglecs-3, 5, $6,7,8,9,10$, and 11) have intracellular domains that bear homology to that of PD- $1^{24}$, including an immunoreceptor tyrosine-based inhibition motif (ITIM) preceding a switch 
motif (ITSM) (Fig. 1a,b Supplementary Fig. 1). These cytosolic ITIM/ITSM domains recruit protein tyrosine phosphatases, ultimately resulting in inhibitory signaling and immune cell suppression ${ }^{25,26}$. Thus, we and others speculated that Siglec engagement of cell-surface sialoglycans has inhibitory consequences similar to PD-1 engagement of PD-L127,28. In support of this hypothesis, Siglec-9 was recently shown to be upregulated on tumor-infiltrating $T$ cells and correlated with reduced survival of cancer patients ${ }^{17}$. Reciprocally, genetic knockout or inhibition of tumor sialic acid synthesis and reduced presence of Siglec ligands enhanced immune infiltration and reduced tumor growth $^{17,29}$. The sialoglycan axis may therefore be a major contributor to tumor immune suppression and an attractive target for cancer immune therapy.
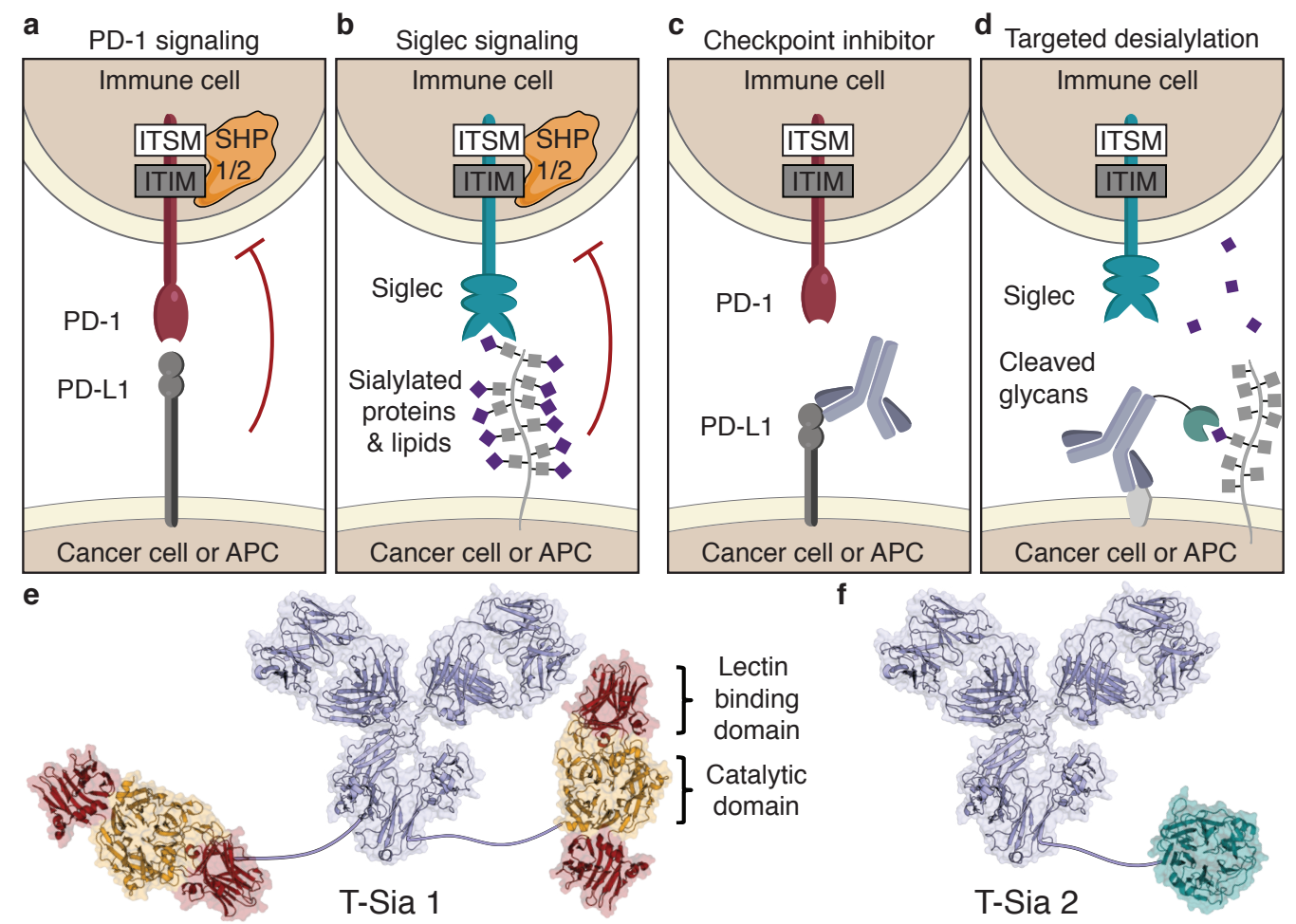

Fig. 1. Targeted desialylation of Siglec ligands with the antibody-enzyme conjugate T-Sia 2 as a modality for immune therapy. a, PD-1 and $\mathbf{b}$, Siglecs are receptors that suppress immune cell function upon ligand binding. Engagement of PD-1 and Siglec receptors leads to recruitment of SHP phosphatases to the cytosolic ITIM/ITSM domains and inhibits immune cells, APC = antigen-presenting cell. c, PD-L1 checkpoint inhibitor therapy uses antibodies to bind PD-L1 and block extracellular interactions to PD-1, inhibiting SHP recruitment and enhancing the immune response to cancer. d, Targeted desialylation with an antibody-sialidase conjugate catalytically removes a chemically diverse 
group of Siglec ligands and prevents SHP recruitment to the Siglec ITIM/ITSM domains. e, Representation of previously reported T-Sia 1 , in which trastuzumab was conjugated to two molecules of $V$. cholerae sialidase. f, Illustration of T-Sia 2, where trastuzumab is linked to an average of one $S$. typhimurium sialidase. Trastuzumab is represented by mouse IgG1 PDB: 1IGY, V. cholerae sialidase: 1WOP, S. typhimurium sialidase: 3SIL.

Sialoglycan ligands exhibit significant chemical heterogeneity and are fused to a wide range of cell-surface protein and lipid scaffolds. Development of ligand-sequestering antibodies (analogous to PD-L1 blockade as illustrated in Fig. 1c) is therefore challenging. Thus, we previously conceived of a therapeutic modality comprising a sialic acid-cleaving enzyme fused to a tumor-targeting antibody (Fig. 1d,e) to catalytically deplete sialoglycans in a tumor-specific manner ${ }^{30}$.

Here, we advance this strategy to full proof-of-concept by demonstrating that selective removal of sialoglycans from cancer cells using antibody-sialidase conjugates can improve the antitumor immune response. These efforts necessitated rational design and screening for optimal sialidase activity to yield Trastuzumab-sialidase conjugate 2 (TSia 2) (Fig. 1f), which exhibits a low off-target activity and high chemical stability needed for in vivo use. In a syngeneic orthotopic HER2 ${ }^{+}$breast cancer model, targeting glycoimmune checkpoints with T-Sia 2 delayed tumor growth and enhanced immune infiltration, leading to prolonged survival of mice with trastuzumab-resistant breast cancer.

\section{Results}

\section{Minimizing off-target sialidase activity}

We previously reported on an antibody-sialidase molecule, T-Sia 1 (Fig. 1e), constructed by conjugation of Vibrio cholerae (VC) sialidase to each trastuzumab heavy chain (chemical conjugation strategy described in Supplementary Fig. 2) ${ }^{30}$. Although TSia 1 efficiently cleaved sialoglycans from HER2 ${ }^{+}$cells at low doses, the conjugate had considerable trastuzumab-independent activity as well ${ }^{30}$, which we ascribed to the low apparent $\mathrm{K}_{\mathrm{M}}$ value of $\mathrm{VC}$ sialidase with polyvalent substrates (including cell surfaces) ${ }^{31}$. 
This attribute reflects the engagement of VC sialidase's two lectin domains (Fig. 1e), which enable cell surface binding independent of antibody targeting ${ }^{31}$.
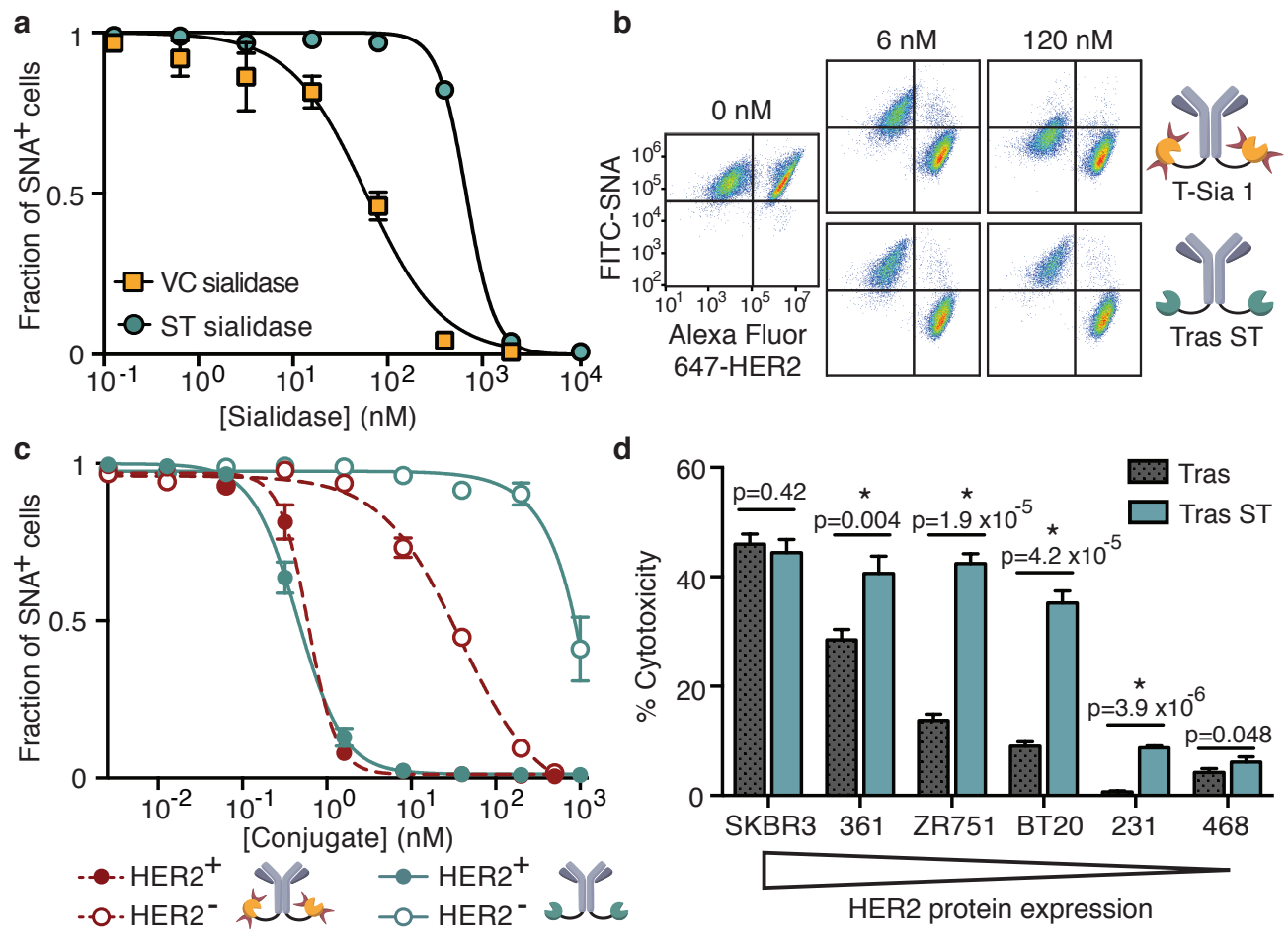

HER2 protein expression

Fig. 2. Salmonella typhimurium sialidase cleaves sialic acid and enhances NK-mediated ADCC of $\mathrm{HER2}^{+}$cells with reduced off-target desialylation of HER2 ${ }^{-}$cells. a, MDA-MB-468 cells were treated with sialidases at various concentrations and stained with SNA-FITC probe. Flow cytometry gating quantified the fraction of $\mathrm{SNA}^{+}$(sialylated) cells and the results were fit to a four-parameter variable slope. b, Representative flow cytometry dot plots of HER2 ${ }^{+}$(SK-BR-3) and HER2- (MDA-MB-468) cells treated with T-Sia 1 or trastuzumab-ST sialidase (tras-ST). c, Fraction of sialylated $\left(\mathrm{SNA}^{+}\right)$cells quantified by flow cytometry gating (as shown in b) after treatment with various concentrations of antibody sialidase conjugates and fit to a variable four-parameter slope. d, Breast cancer cell lines were treated with $10 \mathrm{nM}$ trastuzumab-ST sialidase (tras-ST) or trastuzumab alone (tras). IL-2-activated human NK cells were added at a ratio of 8:1 and NK cell-mediated ADCC was quantified by LDH assay after $4 \mathrm{~h}$. P-values of multiple two tailed $t$ tests are shown with an asterisk indicating significance using the Holm-Sidak multiple comparisons correction at $\alpha=0.05$. Graphs $\mathbf{a}, \mathbf{c}$, and $\mathbf{d}$, represent Mean $\pm S D$ of $n=3$ experimental replicates.

Accordingly, our first goal was to identify a more suitable sialidase from a repertoire that lacks such lectin domains. We expressed six recombinant bacterial and human sialidases in E. coli (Supplementary Fig. 3a). The sialidases were screened for in vitro 
activity and for their ability to enhance natural killer (NK) cell-mediated antibodydependent cellular cytotoxicity (ADCC) against BT-20 breast cancer cells (Supplementary Fig. 3b,c). The Salmonella typhimurium (ST) sialidase $\mathrm{NanH}$ was selected for its relatively high $K_{M}$ value (mM range) against polyvalent targets ${ }^{31-33}$, enhancement of NK cell-mediated ADCC, and stability (Supplementary Fig. 3b-d). To ensure that ST sialidase would cleave Siglec-binding sialoglycans on breast cancer cells, we performed flow cytometry assays using Siglec-9- and -7-Fc fusion proteins as probes. Treatment of nine different breast cancer cell lines with ST sialidase reduced Siglec-9- and $-7-\mathrm{Fc}$ binding signal by $>96 \%$ and $>50 \%$, respectively (Supplementary Fig. $3 \mathrm{e}-\mathrm{g}$ ). As postulated, ST sialidase on its own was less efficient than VC sialidase at removing sialoglycans from the cell surface ( $E_{50}>10$-fold higher) (Fig. 2a).

To determine whether ST sialidase could be rendered selectively active by cell-surface targeting, we conjugated the enzyme to trastuzumab using a similar strategy as previously reported ${ }^{30}$, but in this case the ST sialidase was modified at a recombinantly inserted Cys residue (Supplementary Fig. 4a,b and 5a). The enzymatic activity of the sialidase, as measured with a fluorogenic substrate, was retained after conjugation to trastuzumab (Supplementary Fig. 5b). We next compared the activity and selectivity of the trastuzumab-ST sialidase conjugate to T-Sia 1 in a co-culture assay comprising HER2 ${ }^{+}$SK-BR-3 and HER2 MDA-MB-468 cells. Using the lectin Sambucus nigra agglutinin (SNA) as a probe for cell-surface sialoglycans, both conjugates desialylated HER2 ${ }^{+}$target cells at concentrations near trastuzumab's reported $K_{D}$ of $5 \mathrm{nM}$ (Fig. $\left.2 b\right)^{34}$ by flow cytometry. However, T-Sia 1 caused substantial off-target desialylation of MDAMB-468 cells with an $\mathrm{EC}_{50}$ of $38 \mathrm{nM}$, whereas the trastuzumab-ST sialidase completely abrogated off-target reactivity at that concentration; its $\mathrm{EC}_{50} \sim 1 \mu \mathrm{M}$ (Fig. 2c and Supplementary Fig. 5c). Furthermore, the ST sialidase conjugate retained the ability to enhance NK cell-mediated ADCC of cells expressing medium and low levels of HER2 (Fig. 2d and Supplementary Fig. 6a,b). As expected, the highly trastuzumab-sensitive HER2-high SK-BR-3 cell line delivers a sufficiently strong activating signal to NK cells via FcyRIII and removal of the inhibitory Siglec signal provides no added benefit ${ }^{30}$. In summary, antibody conjugation to a sialidase with low intrinsic binding ability retains on- 
target desialylation activity while increasing the therapeutic window from 60-fold (T-Sia 1) to 2000 -fold.

\section{Optimization of the chemical stability of T-Sia 2}

To enhance the stability of our conjugates and enable in vivo examination, several advances to the construct design were required. Specifically, the oxime bond used in TSia 1 is prone to hydrolysis in biological settings (Supplementary Fig. 2) ) $^{35,36}$. We previously developed the Pictet-Spengler ligation, that forms a stable C-C adduct with SMARTag (aldehyde)-modified proteins ${ }^{35,37}$. More recently, a related process termed the hydrazino-iso-Pictet Spengler (HIPS) reaction was developed ${ }^{38}$. An antibody-HIPSmaytansinoid conjugate demonstrated a long serum half-life and had the highest tolerated dose of any maytansinoid antibody-drug conjugate reported in monkeys ${ }^{39}$, enabling advancement into human clinical studies ${ }^{40}$. Based on these precedents, we synthesized a HIPS-azide linker (1) (Supplementary Fig. 7) and conjugated this to SMARTag-labeled trastuzumab with full conversion detected by mass spectrometry (Fig 3a and Supplementary Fig. 8a-c). We confirmed the enhanced stability of trastuzumab conjugated to HIPS over oxime in human plasma and on live cells (Supplementary Fig. S9a-e).

To improve the uniformity of the trastuzumab-ST conjugate, we selectively and stably alkylated the engineered C-terminal Cys residue of ST sialidase with an $\alpha$ chloroacetamide-DBCO linker (2) (Fig. 3a and Supplementary Fig. 10) under mild reducing conditions (Supplementary Fig. 11a). We confirmed that the engineered Cys residue was uniquely modified, with no off-target reactivity towards the four endogenous ST sialidase Cys residues (Supplementary Fig. 11b-d). Finally, trastuzumab-azide and ST sialidase-DBCO were coupled by copper-free click chemistry ${ }^{41}$ to produce T-Sia 2 (Fig. 3a).

To determine the optimal enzyme/antibody ratio (EAR) of the conjugate, we isolated TSia 2 fractions with EARs of $\sim 1$ and $\sim 2$ by size exclusion chromatography (Supplementary Fig. 12a). In NK cell-mediated ADCC assays, T-Sia 2 with an EAR $\sim 1$ 
outperformed EAR $\sim 2$ by a small but significant margin (1.13-fold increase in cytotoxicity, $p=0.035$ ) (Supplementary Fig. 12b-c). This improvement may result from enhanced FcyRIII binding to a less sterically hindered epitope. Accordingly, we optimized our conjugation procedure to increase the proportion of T-Sia 2 with an EAR $\sim$ 1. We characterized the conjugate by SDS-PAGE (Fig. 3b) and mass spectrometry (Supplementary Fig. 13a) and determined an EAR $=0.9$ by HPLC analysis (Fig. $3 c$ and Supplementary Fig. 13b). We confirmed that T-Sia 2 binds its antigen HER2 similarly to trastuzumab (Supplementary Fig. 13c), and also enhanced NK cell- and yd T cellmediated ADCC compared to trastuzumab (Supplementary Fig. 14a-c).

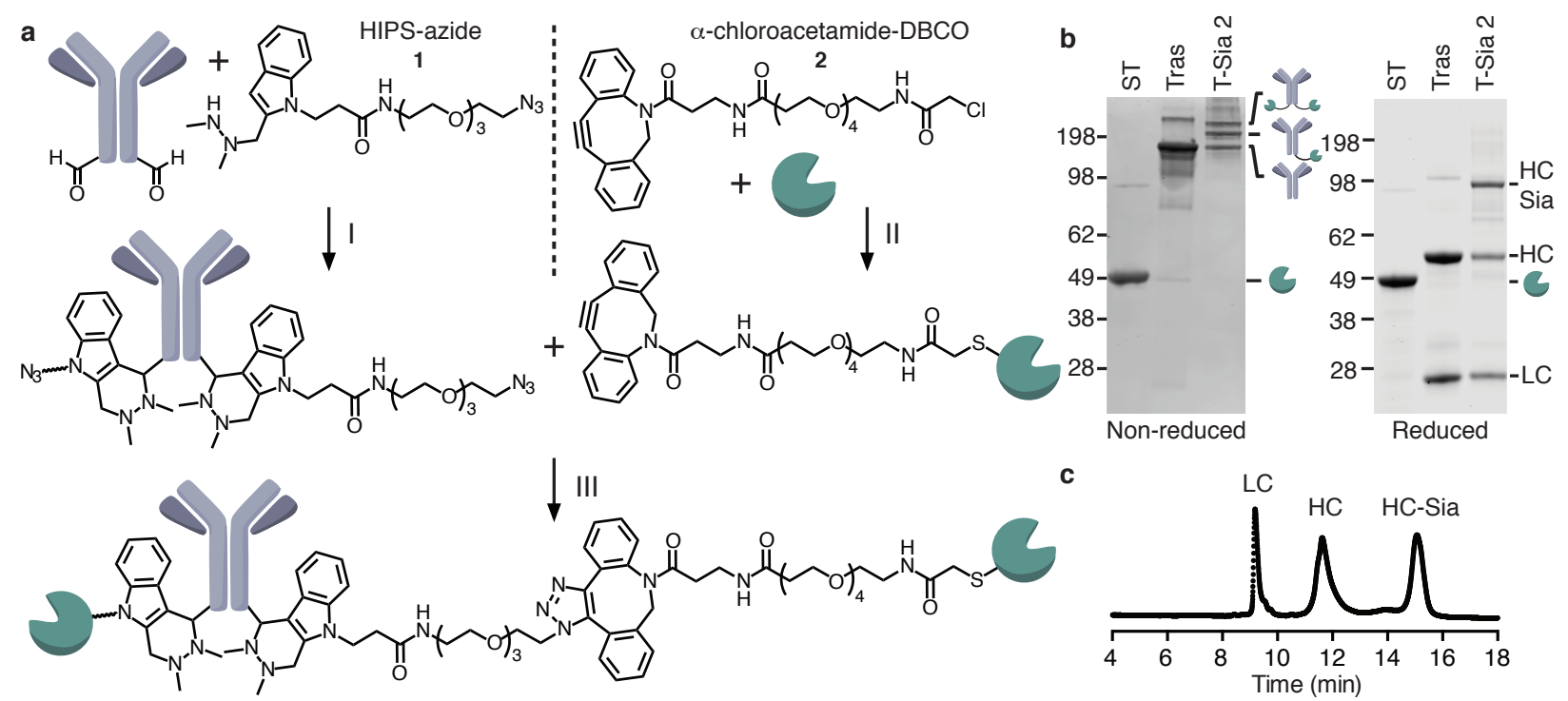

Fig. 3. Synthesis of T-Sia 2. a, T-Sia 2 scheme: (I) Trastuzumab with heavy-chain formylglycine residues was reacted with 20 equiv. HIPS-azide 1 in citrate buffer pH 5.5. (II) ST sialidase was siteselectively reacted with 20 equiv. a-chloroacetamide-DBCO 2 under mildly reducing conditions. (III) Sialidase-DBCO and trastuzumab-azide were coupled via copper-free click chemistry to form T-Sia 2 . b, SDS-PAGE with non-reducing buffer (left) and reducing buffer (right) of sialidase-DBCO, trastuzumabHIPS-azide, and T-Sia 2. c, Representative HPLC trace of T-Sia 2. Abbreviations: LC = antibody light chain, $\mathrm{HC}=$ antibody heavy chain, $\mathrm{HC}-\mathrm{Sia}$ = antibody heavy chain conjugated to ST sialidase.

T-Sia 2 delays tumor growth in the trastuzumab-resistant syngeneic EMT6 breast cancer model 
To test the efficacy of T-Sia 2 in vivo, we selected the syngeneic orthotopic mouse EMT6 mammary carcinoma model ${ }^{42}$, in which the EMT6 cell line was engineered to express HER2 yet remained resistant to trastuzumab monotherapy in mice ${ }^{43}$. In vitro, T-Sia 2 cleaved sialoglycans from HER2 ${ }^{+}$EMT6 cells, causing a decrease in binding to both human and mouse Siglec-Fc fusion probes (Supplementary Fig. 15), and enhanced human NK cell-mediated ADCC (Fig. 4a).

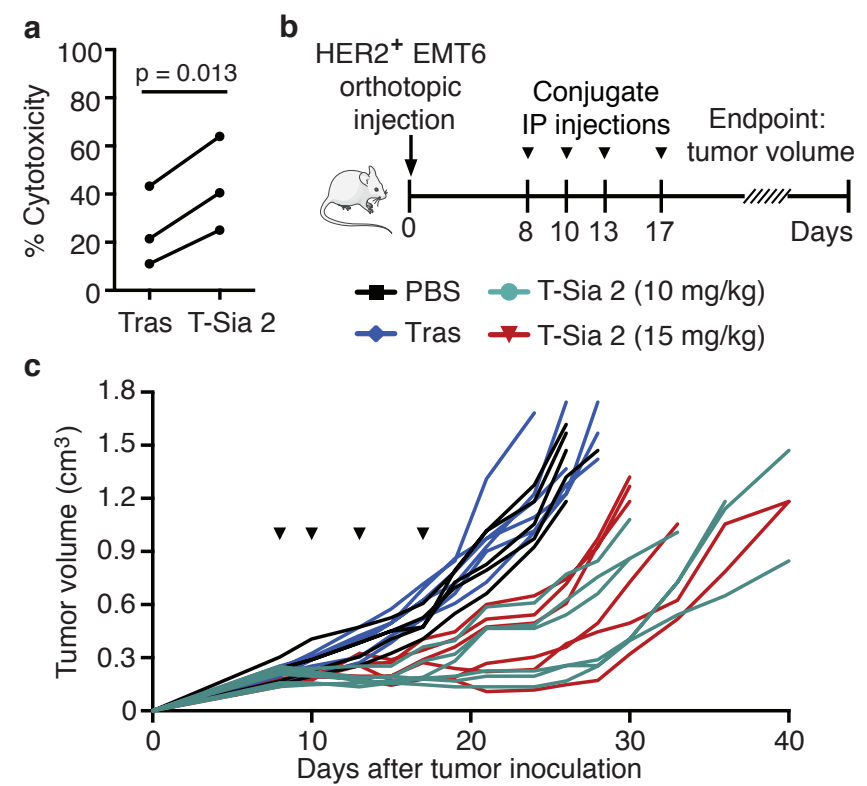

Fig. 4. T-Sia 2 delays tumor growth in a HER2 $^{+}$EMT6 syngeneic mouse breast cancer model that is resistant to trastuzumab alone. a, HER2 ${ }^{+}$EMT6 cells were treated with PBS, $10 \mathrm{nM}$ trastuzumab (Tras), or $10 \mathrm{nM} \mathrm{T-Sia} \mathrm{2.} \mathrm{Human} \mathrm{NK} \mathrm{cells}$ were added at a ratio of $3: 1$ and NK cellmediated ADCC was quantified by flow cytometry after $4 \mathrm{~h}$ and subtracted from PBS controls. A paired $t$ test is shown for three biological replicates using different NK cell donors. b, HER2 ${ }^{+}$EMT6 cells were injected into the mammary fat pad of Balb/c mice at day
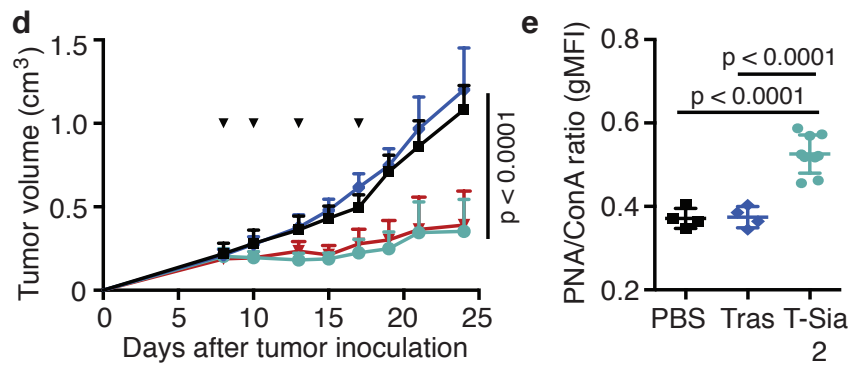
0 . At day 8, mice were injected intraperitoneally (IP) with PBS $(n=5)$, trastuzumab $(15 \mathrm{mg} / \mathrm{kg}, \mathrm{n}=6)$, or T-Sia $2(10$ $\mathrm{mg} / \mathrm{kg}$ or $15 \mathrm{mg} / \mathrm{kg}, \mathrm{n}=6$ ). Tumor volume was measured over time. c, Individual growth curves of the HER2 ${ }^{+}$EMT6 tumors in mice. d, Mean + SEM tumor volume over time for animals described in b, RM two-way ANOVA comparing trastuzumab to T-Sia $2(10 \mathrm{mg} / \mathrm{kg})$. e, Lectin stain with PNA to detect exposed galactose of extracted tumor cells from mice in $\mathbf{b}$. Geometric mean $(\mathrm{gMFI}) \pm \mathrm{SD}$ PNA/ConA, $\mathrm{n}=4$ (PBS and tras) and $\mathrm{n}=9$ (T-Sia 2, both doses), ordinary one-way ANOVA with Tukey's multiple comparison.

Next we performed in vivo experiments as depicted in Fig. 4b. HER2 ${ }^{+}$EMT6 cells were injected into the mammary fat pads of mice followed by intraperitoneal (IP) treatment 
with PBS, trastuzumab, or T-Sia 2. After 28 days, all mice in the PBS or trastuzumab treated groups had reached a tumor burden requiring sacrifice (Supplementary Fig. 16a). In contrast, T-Sia 2 at both doses extended mouse survival to 40 days and demonstrated significant tumor growth delay compared to trastuzumab alone (Fig. 4c,d and Supplementary Fig. 16a). No significant difference in tumor growth was observed between the two doses of T-Sia 2, and although the $10 \mathrm{mg} / \mathrm{kg}$ dose of T-Sia 2 was well tolerated, the $15 \mathrm{mg} / \mathrm{kg}$ dose resulted in a minimal weight loss in mice compared to untreated controls at day 24 (Supplementary Fig. 16b). Lectin staining of tumor suspensions with Peanut Agglutinin (PNA), which binds to galactose residues exposed upon sialidase treatment, showed increased labeling in the T-Sia 2-treated tumors compared to the PBS- or trastuzumab-treated mice 2-4 weeks after the final conjugate injections (Fig. 4e). These data indicate that T-Sia 2 desialylated the tumor microenvironment (TME) and delayed EMT6 tumor growth in mice.

To determine where T-Sia preferentially accumulates in mice, we modified the conjugate with IRDye $800 \mathrm{CW}$ NHS ester and imaged mice bearing subcutaneous EMT6 tumors treated with $500 \mathrm{pmol}$ conjugate $(\sim 4 \mathrm{mg} / \mathrm{kg}), 100 \mathrm{pmol}$, and $20 \mathrm{pmol}$, and PBS control. After 48 hours, fluorescent dye clearly localized to the tumors on the left flank of the mice at the highest dose (Supplementary Fig. 17a). After 4 days, mice were sacrificed to further analyze T-Sia localization in individual organs. Both the 500 and 100 pmol doses of 800-labeled T-Sia 2 significantly accumulated in tumors, however the higher dose of 500 also had significant fluorescent accumulation in the liver and kidneys, likely involved in antibody and fluorophore clearance (Supplementary Fig. 17bd). Interestingly, all doses exhibited significant desialylation of the tumors analyzed by flow cytometry after 4 days by MALII and PNA lectin staining (Supplementary Fig. 17e).

\section{Sialidase activity, HER2 targeting, and FcyR binding contribute to T-Sia 2 activity in mice}


a
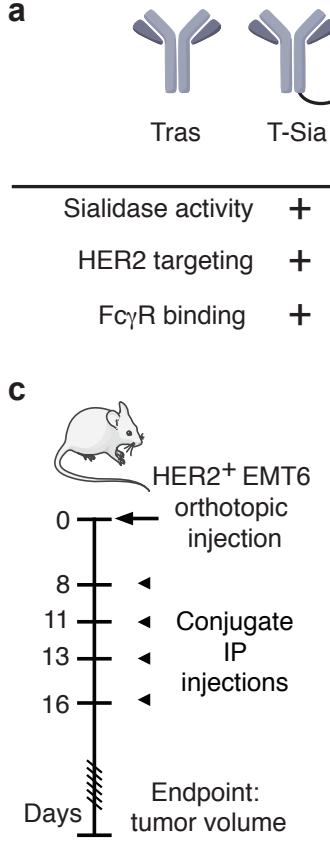

Days tumor volume

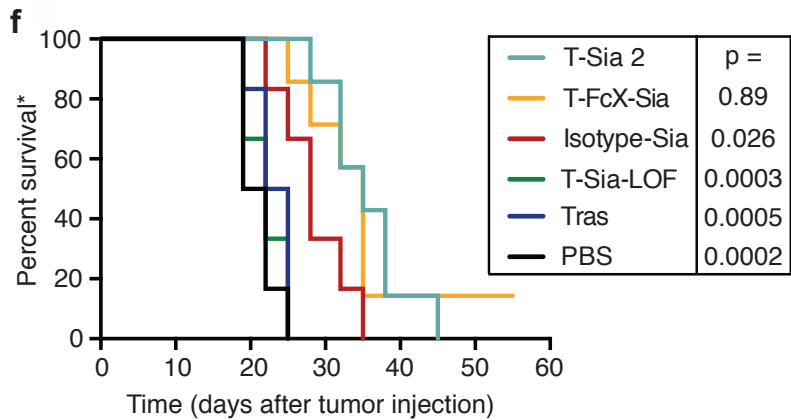

d b
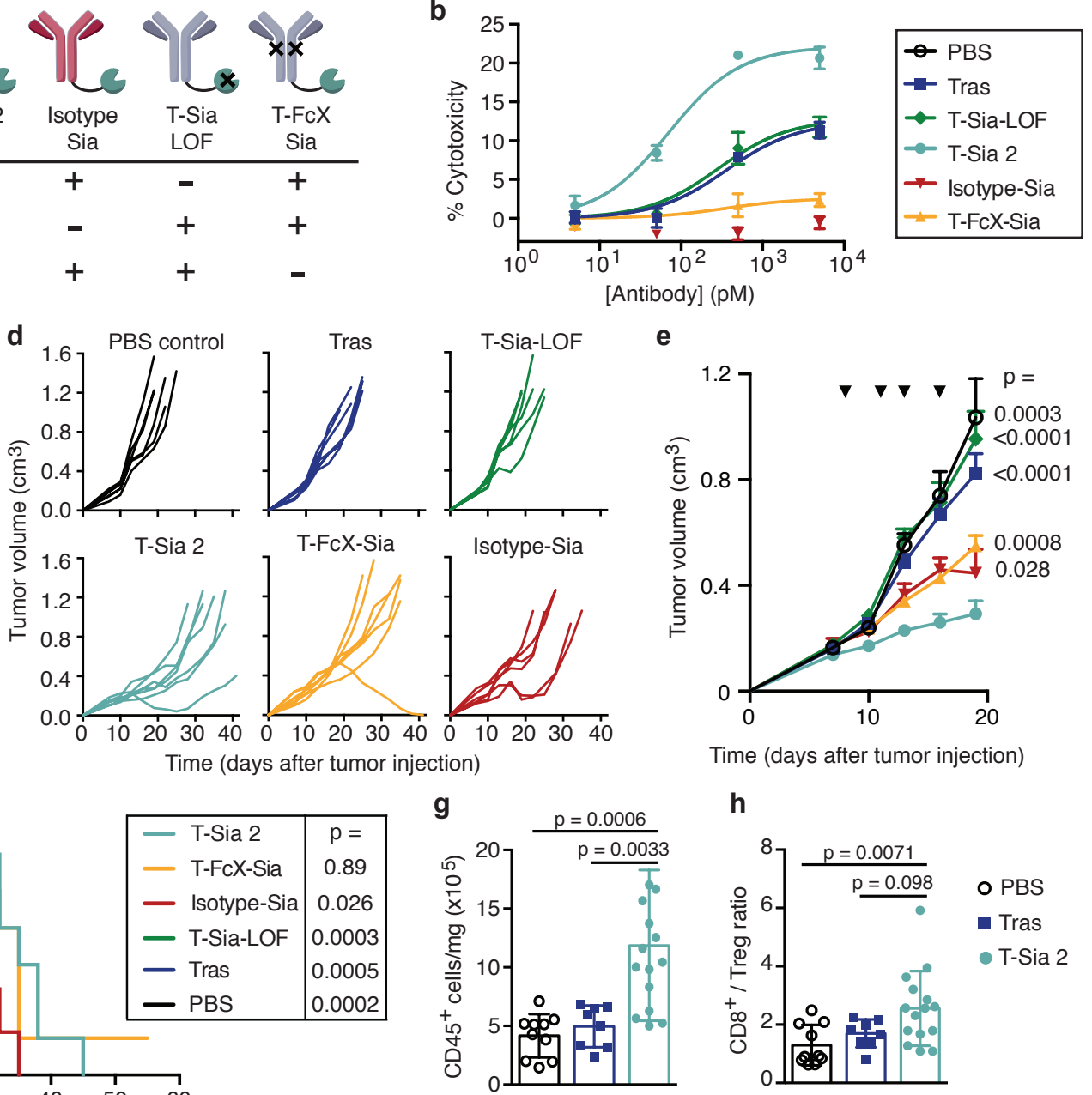

e

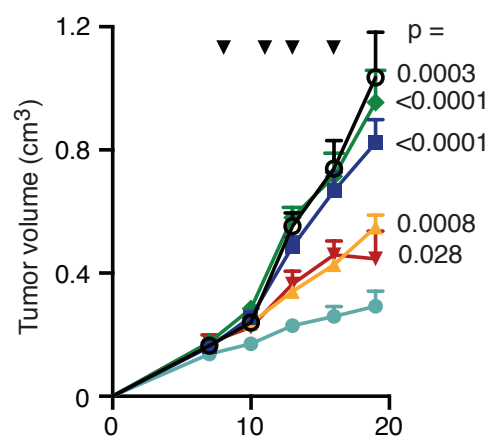

Time (days after tumor injection)

h

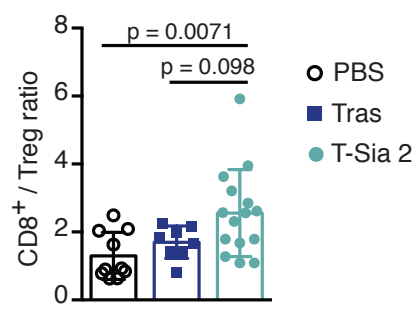

Fig. 5. HER2-targeting and FcYR engagement are important mechanisms of T-Sia 2 therapy. a, Schematic of the antibody-sialidase conjugates. b, NK cell-mediated ADCC of ZR-75-1 cancer cells treated with antibody conjugates, $(n=3), E / T=3$, detecting percent cytotoxicity by LDH release after $8 \mathrm{~h}$. c, HER2 ${ }^{+}$EMT6 cells $\left(1 \times 10^{6}\right)$ were injected into the mammary fat pad of Balb/c mice. On day 8 , mice were injected intraperitoneally with PBS $(\mathrm{n}=6)$, or $10 \mathrm{mg} / \mathrm{kg}$ trastuzumab (tras) $(\mathrm{n}=6)$, T-Sia-LOF $(\mathrm{n}=6)$, Isotype-Sia $(n=6)$, T-FcX-Sia $(n=7)$, or T-Sia $2(n=7)$. d, Individual tumor growth curves for mice in c. e, Mean + SEM of tumor growth from mice in c; RM two-way ANOVA of treatments compared to T-Sia 2. $\mathbf{f}$, Kaplan-Meier plot of the time-to-sacrifice of mice in c; with Log-rank Mantel-Cox tests compared to T-Sia 2. g, Total tumor-infiltrated leukocyte count normalized to tumor weight. $\mathbf{h}$, Ratio of $\mathrm{CD}^{+} \mathrm{T}_{\text {cells }}$ to $\mathrm{T}_{\text {reg }}$ cells. i, \%CD69 ${ }^{+}$of NK cells. Leukocyte analysis quantified from the two independent mouse experiments (Figs 4 \& 5); ordinary one-way ANOVA with Dunnet's multiple comparisons to T-Sia 2, $n=8-15$ mice/group, mean $\pm S D$. 
To better understand the mechanisms underlying T-Sia 2's antitumor activity, we synthesized three control molecules using the same conjugation strategy as with T-Sia 2 (Fig. 5a). The first was T-Sia 2 in which the sialidase's catalytic nucleophile was mutated $(Y \rightarrow A)$, causing loss of function (T-Sia-LOF) (Supplementary Fig. 18a-c). The second was an isotype control human IgG1-sialidase conjugate with the antibody motavizumab that targets the respiratory syncytial virus, which has $87 \%$ identity to trastuzumab (Isotype-Sia) (Supplementary Fig. 19a-f) ${ }^{44,45}$. The third was a variant possessing several point mutations (ELLG $\rightarrow$ PVA-) in the FcyR binding domain of trastuzumab, which abolishes most effector FcyR and CDC interactions on immune cells yet maintains the majority of the neonatal $F c$ receptor ( $F c R n)$ binding and therefore native antibody recycling (T-FcX-Sia) (Supplementary Fig. 20a-f $)^{46,47}$. NK cell-mediated ADCC assays with several HER2 ${ }^{+}$cell lines demonstrated that T-Sia 2 was superior in eliciting cell death whereas T-Sia-LOF had comparable activity to trastuzumab alone (Fig. 5b and Supplementary Fig. 21). As expected, Isotype-Sia was ineffective in stimulating ADCC against HER2 ${ }^{+}$cells and T-FcX-Sia had greatly diminished FcyRIIImediated ADCC activity compared to T-Sia 2 in vitro.

The antibody-sialidase conjugates were then injected intraperitoneally into Balb/c mice growing HER2 ${ }^{+}$EMT6 tumor cells in their mammary fat pads (scheme in Fig. 5c). For PBS-, trastuzumab-, and T-Sia 2-treated mice, the tumor growth curves were consistent with the previous mouse experiment: tumors in PBS- and trastuzumab-treated mice grew quickly, while tumors in T-Sia 2-treated mice exhibited delayed growth (Fig. $5 \mathrm{~d}$ and Supplementary Fig. 22a). T-Sia-LOF-treated mice had tumor growth that was indistinguishable from trastuzumab- and PBS-treated mice, indicating that sialidase activity was necessary for therapeutic effect (Fig. 5d,e and Supplementary Fig. 22b). TSia 2-treated mice showed an early delay in tumor growth that was not evident in TFcX-Sia-treated mice (Fig. 5e and Supplementary Fig. 22b); however, there was no significant difference in time to sacrifice between the two treatment groups (Fig. 5f). These data suggest that FcyR binding or CDC function may be more important in the early phases of the antitumor response, and that T-Sia 2 is potentiating both FcyRdependent and -independent immune responses. 
We observed a delay in tumor growth in mice treated with the Isotype-Sia construct when compared to untreated, trastuzumab, or T-Sia-LOF controls (Fig. 5e). It is possible that systemic exposure to sialidase activity potentiates immune cell reactivity against the tumor through a more general loss of immunomodulatory sialoglycans. Alternatively, the dose may be high enough to permit some desialylation of the tumor (Supplementary Fig. 22c). Nonetheless, targeting the sialidase activity to the tumors through conjugation to the trastuzumab antibody enhanced the therapy significantly; TSia 2 was more effective at prolonging the time to sacrifice than Isotype-Sia (Fig. 5f) and Isotype-Sia-treated tumors grew more rapidly than the HER2-targeted conjugate (Fig. 5e and Supplementary Fig. 22b).

To further investigate effects of sialidase exposure, we analyzed blood cell counts pursuant to administration of the above antibody-sialidase conjugates. Forty-eight hours after the first injection of conjugates, red and white blood cell counts were comparable to PBS-treated controls (Supplementary Fig. 22d,e) whereas the platelet count decreased to an average of 157 platelets $/ \mathrm{nL}$ blood in the mice treated with any construct containing active sialidase (Supplementary Fig. 22f), likely mediated by the asialoglycoprotein receptor in the liver, which binds to exposed galactose upon desialylation ${ }^{48,49}$. Thrombocytopenia is an unfortunate but common symptom of cancer treatment and some platelet loss may be a favorable prognostic biomarker of $\mathrm{ICl}$ therapy ${ }^{50}$. None of the sialidase conjugates instigated weight loss or signs of ill health in treated mice (Supplementary Fig. 22g).

\section{T-Sia 2-treatment enhanced activated immune cell infiltration}

Tumors of untreated, trastuzumab, and T-Sia 2-treated mice were collected upon sacrifice and the infiltrating immune cells were extracted and analyzed by flow cytometry. The results from the combined mouse experiments showed an increase in total tumor leukocytes in the T-Sia 2-treated mice compared to untreated- and trastuzumab-treated mice (Fig. $5 \mathrm{~g}$ ). We also observed a general increase of tumorinfiltrating lymphocytes (TILs) (Supplementary Fig. 23). This is encouraging as studies 
have shown that high TILs following treatment of breast cancer patients are a favorable prognostic marker for overall and metastasis-free survival ${ }^{51,52}$. Additionally, T-Sia 2 treatment augmented the ratio of CD8+ $T$ cells to $T_{\text {reg }}$ cells (Fig. $5 \mathrm{~h}$ ), another indicator of improved patient prognosis in breast cancer ${ }^{53}$.

Other immune marker changes in the T-Sia 2-treated tumors included an increase in MHCII+ tumor associated macrophages (TAMs) and a decrease in CD206+ TAMs (Supplementary Fig. 23), indicating a potential switch to a more inflammatory macrophage polarization ${ }^{54}$. Additionally, T-Sia 2-treated tumors demonstrated an increase in activated (CD69+, Fig. 6c,d) and cytotoxic (granzyme b+, Fig. 6e,f) CD8 ${ }^{+} \mathrm{T}$

and NK cells ${ }^{55,56}$. Analysis of other immune cell populations showed trends suggesting enhanced infiltration of dendritic- and B-cells (Supplementary Fig. 23). In summary, a more infiltrated and activated tumor immune microenvironment could be observed with activation of both innate and adaptive immune cells.

\section{T-Sia 2 does not delay tumor growth in a Siglec-E knockout mouse tumor model}

Recent evidence suggests that Siglec-E (a Siglec-7/-9 homolog) is the major Siglec present on the tumor infiltrating T cells of several cancer models including the EMT6 tumor model in mice ${ }^{17}$. We therefore hypothesized that Siglec-E might be a major contributor to the immunosuppressive phenotype in these tumors and that destroying Siglec-E ligands is an important mechanism of action of T-Sia 2 in mice. As there are no known fully antagonistic blocking antibodies for Siglec-E, we decided to test our TSia 2 conjugate in mice lacking inhibitory Siglec-E $\left(S i g-\mathrm{E}^{-l}\right)$, a mouse model with the C57BL/6 background developed by the Crocker lab ${ }^{57}$. 


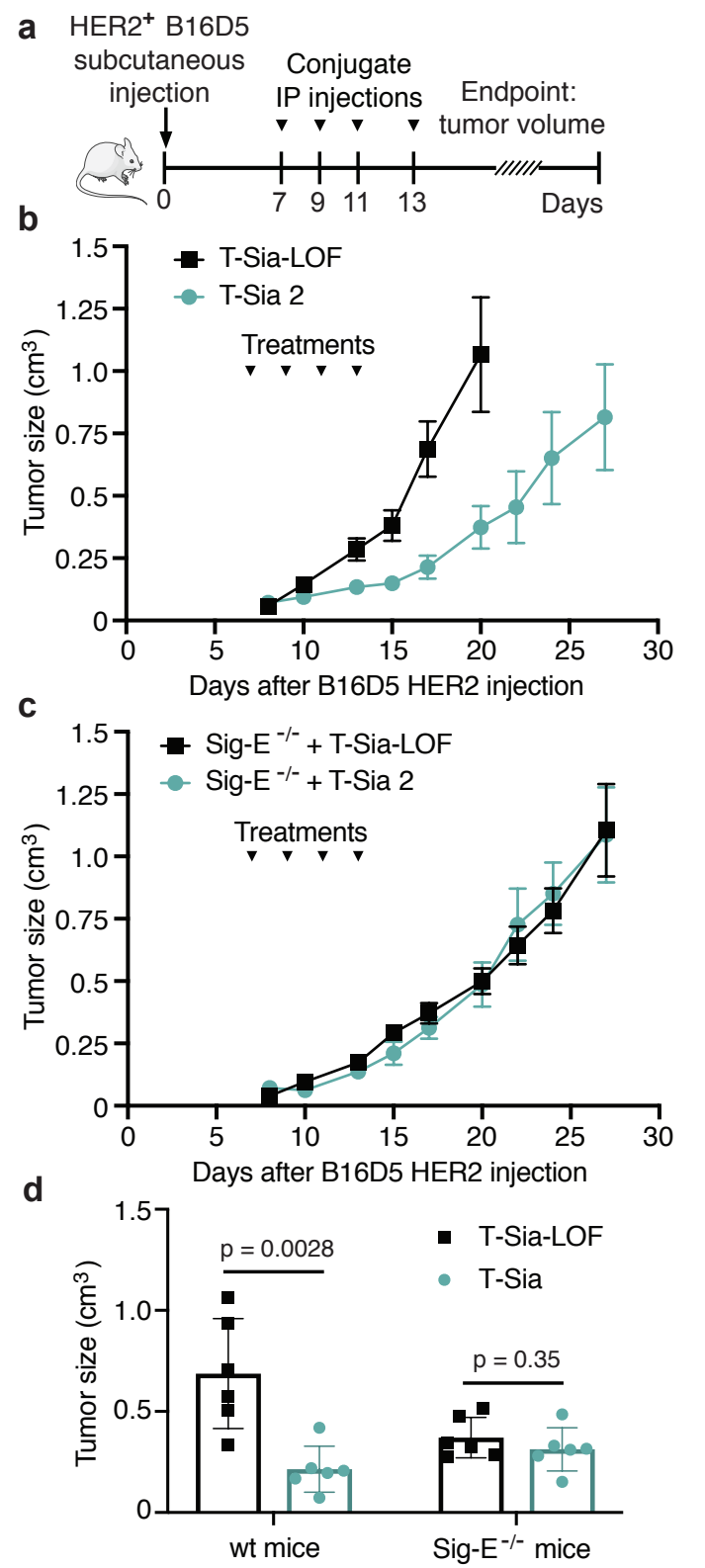

Fig. 6. T-Sia 2 therapeutic effect is dependent on functional Siglec-E in mice. a, HER2 ${ }^{+}$B16D5 cells $\left(5 \times 10^{5}\right)$ were injected subcutaneously into either $\mathbf{b}$, wt C57BL/6 mice or c, Sig- $\mathrm{E}^{-/-} \mathrm{C} 57 \mathrm{BL} / 6$ mice. On day 7 , mice were injected intraperitoneally with T-Sia 2 or the inactive T-Sia-LOF ( $n=6$ mice per group). Tumor size was plotted for all surviving mice until a group reached $n<4$ surviving mice. $d$, wt and Sig- $\mathrm{E}^{-/-}$mouse tumor volumes were plotted and analyzed by unpaired $t$ test on day 17 , the final day with $n=6$ surviving mice for each group.

To do this, we used HER2 ${ }^{+}$B16D5 melanoma tumors, which expressed Siglec ligands that were cleavable by ST sialidase (Supplementary Fig. 24), and injected them subcutaneously into the flanks of either wt or Sig- $\mathrm{E}^{-/-}$C57BL/6 mice, after 7 days, mice were treated with T-Sia 2 or the T-Sia-LOF control conjugate, and tumor growth was assessed over time (Fig. 6a). As expected, the T-Sia 2 molecule delayed tumor growth in wt C57BL/6 mice compared to T-SiaLOF (Fig. 6b). Interestingly, in Sig- $\mathrm{E}^{-/-}$mice there was no benefit of T-Sia 2-treatment over the loss-of-function sialidase conjugate (Fig. $6 \mathrm{c}, \mathrm{d}$ ), indicating that the benefit of targeted sialidase activity in vivo is dependent on functional Siglec-E in this mouse model.

\section{Discussion}

The correlation of hypersialylation with cancer progression was first reported in the $1960 s^{58}$. In the 1970s, researchers explored the effects of sialidase treatment for cancer therapy, but without the framework for a cogent mechanistic hypothesis and with mixed results likely due to rapid recovery of cell-surface sialic $\operatorname{acid}^{58}$. The development of modern techniques for bioconjugation, as well as recent breakthroughs in our 
understanding of the role of sialoglycans in numerous pathways of immune suppression prompted us to explore whether tumor-targeted sialidase enzymes could now be effectively harnessed for cancer therapy. These efforts yielded T-Sia 2, a trastuzumabS. typhimurium sialidase conjugate that significantly delayed tumor growth in a trastuzumab-resistant cancer model. The use of two bioorthogonal chemistry tools - a HIPS reaction with aldehyde-tagged antibodies and copper-free click chemistry enabled modular assembly of conjugates using common functionalized antibody and enzyme moieties. Key factors in the performance of T-Sia 2 were its target specificity and plasma stability imparted by antibody conjugation, which facilitated desialylation of the tumor microenvironment that was measurable 2-4 weeks after conjugate injection.

T-Sia 2-treatment enhanced the $\mathrm{CD}^{+} \mathrm{T}$ cell/ $\mathrm{T}_{\text {reg }}$ ratio in the tumors and showed an increase in activated and cytotoxic $\mathrm{NK}$ and $\mathrm{CD} 8^{+} \mathrm{T}$ cells. These results are consistent with literature that implicates NK cells ${ }^{15,16}$ and $\mathrm{CD}^{+} \mathrm{T}^{\mathrm{T}}$ cells ${ }^{29}$ as key mediators of the immune response against hyposialylated tumor cells. Much of T-Sia 2's anticancer activity appears to be mediated by the removal of ligands for immune-suppressing mouse Siglec-E receptor. Several human Siglecs have now been implicated as immune suppressors in the TME, including Siglecs 7, 9, and $15^{17,27,59,60}$, but the importance of their relative contributions may vary from tumor to tumor. Although the molecular details of their specific biological ligands remain an active area of research, these Siglec family members all require terminal sialoglycans for ligand recognition, and the chemical diversity and complexity of these sialic acids has made it challenging to generate targeted therapeutics with broadly neutralizing activity. The antibody-sialidase conjugates exemplified herein can destroy such sialoglycans in a Siglec-agnostic manner. Further study of Siglec-sialoglycan biology in a range of different cancer subtypes will be essential to elucidate the precise indications where sialidase conjugates like T-Sia 2 may be most effective. Significantly, the modular chemical synthesis of T-Sia 2 can be readily adapted to other FDA-approved therapeutic monoclonal antibodies, allowing for simple and rapid targeting of sialidase enzymes to a wide range of tumor types. 
While this study demonstrates the efficacy of T-Sia 2 as a monotherapy, there is significant future scope for combining antibody-sialidase molecules that target glycoimmune checkpoints with more traditional ICl therapies or antibody-drug conjugates (ADCs). Siglec receptors are expressed across a broad range of innate and adaptive immune cells and Siglec- $9^{+}$CD $8^{+}$TILs co-express immune checkpoint inhibitors ${ }^{17}$, indicating that T-Sia 2 may synergize with immune checkpoint agents. Recent evidence also suggests that removal of surface sialic acid increases the internalization rate of $A D C s$, indicating that targeted desialylation could enhance their cytotoxic effects ${ }^{61}$. We anticipate that glycan-editing antibody therapies will prove a potent tool amongst the wider arsenal of new anticancer therapies moving towards clinical translation.

\section{Methods}

\section{Statistical Analysis}

All statistical analyses were performed using GraphPad Prism 6. P-values are reported for t-tests. For multiple $t$ tests, an asterisk is used to indicate statistical significance after correction for multiple comparisons using the Holm-Sidak method with $\alpha=0.05$. For one-way ANOVA, $p$-values of the ANOVA are reported if $p>0.05$; if $p<0.05$, post-hoc statistics are reported (Tukey's or Dunnet's) as multiplicity-adjusted p-values. The $p$ values of ordinary and RM two-way ANOVA analyses are reported on the figure or in the figure legend; in Supplementary Fig. 8 and 16, post-hoc analyses were performed after two-way ANOVA with Sidak's and Dunnet's multiple comparisons, respectively, and are reported in the figures. For mouse survival curve analysis, a log-rank (MantelCox) test was used to compare between treatment groups.

\section{General synthetic chemistry procedures}

All chemical reagents were purchased in reagent grade from commercial suppliers including Sigma-Aldrich, Thermo Fisher Scientific, TCl, and BroadPharm and used without purification. Unless stated otherwise, all chemical reactions were performed in standard, oven-dried glassware fitted with a rubber septa under an inert atmosphere of nitrogen using anhydrous solvents. Stainless steel syringes were used to transfer airand moisture-sensitive liquids. Anhydrous solvents (acetonitrile (ACN), diethyl ether, 
dichloromethane, $\mathrm{N}, \mathrm{N}$-dimethylformamide, and tetrahydrofuran) were prepared by passing the solvent through an activated alumina column. See supplemental methods for detailed synthetic procedures and characterization of compounds.

\section{Human cell lines}

Cell media, PBS, DPBS, and serum were purchased from Corning Mediatech unless otherwise specified. SK-BR-3, ZR-75-1, and HCC-1954 were purchased from the American Type Culture Collection (ATCC) and cultured in filtered RPMI media 10\% heat-inactivated FBS and no added antibiotics. BT-20, MDA-MB-468, MDA-MB-361, MDA-MB-231, MCF-7, and MDA-MB-453 were purchased from ATCC and cultured in DMEM media containing 10\% heat-inactivated FBS and no added antibiotics. The HEK-Blue cell line for endotoxin detection was thawed into growth medium (DMEM + $10 \%$ HI FBS + Penicillin-Streptomycin $+1 \mathrm{X}$ Normocin) and cultures were maintained in growth medium + 1X HEK-Blue selection. Cultures were grown in T25 and T75 flasks (Thermo Fisher) and maintained at $37{ }^{\circ} \mathrm{C}$ with $5 \% \mathrm{CO}_{2}$. All cultures tested negative for mycoplasma infection by the Lonza MycoAlert Mycoplasma Detection Assay.

\section{Mouse cell lines}

EMT6 cells expressing the HER2 protein were a generous gift from the Zippelius lab (University of Basel, Switzerland) and were cultured in Waymouth's MB 752/1 medium (Thermo Fisher) $+15 \%$ heat-inactivated FBS without antibiotic. B16D5 melanoma tumors expressing HER2 were cultured in DMEM supplemented with $10 \%$ FBS, $1 \%$ gluatamine, $1 \%$ sodium pyruvate, and $1 \%$ amino acids.

\section{Human NK and T cell isolation procedure}

LRS chambers were obtained from healthy anonymous blood bank donors and isolated using Ficoll-Paque (GE Healthcare Life Sciences, GE-17-1440-02) density gradient separation. Cells were cultured in X-VIVO 15 media (Lonza, 04-418Q) supplemented with $5 \%$ heat-inactivated human male $A B$ serum (Sigma Aldrich, H4522). For some experiments, recombinant carrier-free IL-2 (Biolegend, 1:2000 dilution, 589104) was added to further activate NK cells overnight. After 12-24 h, NK cells were isolated from 
PBMCs by immunomagnetic negative selection using the EasySep ${ }^{\text {TM }}$ Human NK Cell Enrichment Kit (STEMCELL Technologies Catalog \# 19055) according to the manufacturer's protocol. Human $\gamma \delta T$ cell were isolated from PBMCs by immunomagnetic negative selection using the EasySep ${ }^{\mathrm{TM}}$ Human Gamma/Delta T Cell Isolation Kit (STEMCELL Technologies, 19255) according to the manufacturer's protocol.

\section{Protein gels and protein concentration}

Protein concentration was determined from the absorbance at $280 \mathrm{~nm}$ using the NanoDrop 2000 Spectrophotometer (Thermo Fisher Scientific) divided by the molar extinction coefficient calculated from protein sequence using ExPASy server (provided by the Swiss Institute of Bioinformatics) ${ }^{62}$. SDS-PAGE protein gels were run on 18 well 10\% Criterion XT Bis-Tris Protein Gels (Bio-Rad, 3450112), 180 V, 40 min -1 h. Running buffer $(2 x)$ for protein gels was made in-house and contained: $4 \%$ sodium dodecyl sulfate (Millipore Sigma L3771), 0.004\% bromphenol blue (Sigma-Aldrich B0126), 20\% glycerol (Chem-Impex 00599), and $120 \mathrm{mM}$ Tris $\mathrm{HCl}$ (Sigma-Aldrich T5941), pH 6.8. For reduced gels, SDS page buffer additionally contained $10 \% 2-$

mercaptoethanol, and proteins in $1 \mathrm{x}$ running buffer were heated in buffer to $95{ }^{\circ} \mathrm{C}$ for 5 min before loading onto gels. Protein gels were stained with AcquaStain Protein Gel Stain (Bulldog Bio AS001000) and imaged on a LI-COR Odyssey CLx imaging system. Protein sequences are reported in the supplementary methods

\section{General DNA procedures and instrumentation}

DNA gBlocks were ordered from Integrated DNA Technologies (IDT). DNA primer sequences were ordered from IDT or Elim Biopharmaceuticals. Plasmids were sequenced by ELIM Biopharmaceuticals and analyzed using SnapGene 3.3.3. PCR was performed in the $\mathrm{C} 100$ Touch Thermal Cycler from Bio-Rad. Unless otherwise specified, PCR amplification was performed using the CloneAmp HiFi PCR premix (Takara) with the following conditions. 


$\begin{array}{lll}\text { PCR conditions } & \text { Amount to add } & \text { Final concentration } \\ \text { 2x HiFi Polymerase } & 12.5 \mu \mathrm{L} & 1 \mathrm{x} \\ \text { Primer 1 } & 5 \mathrm{pmol} & 0.2 \mu \mathrm{M} \\ \text { Primer 2 } & 5 \mathrm{pmol} & 0.2 \mu \mathrm{M} \\ \text { Template DNA } & 10-100 \mathrm{pg} & \\ \text { Water } & \text { Add to } 25 \mu \mathrm{L} \\ & \\ 95^{\circ} \mathrm{C} & 10 \mathrm{~s} \\ 55^{\circ} \mathrm{C} & 10 \mathrm{~s} \\ 72^{\circ} \mathrm{C} & 40 \mathrm{~s} \text { (repeat steps 1-3, 29x) } \\ 72^{\circ} \mathrm{C} & 5 \mathrm{~min}\end{array}$

Amplified DNA was purified by agarose gel: 1\% agarose (Thermo Fisher 16500500) in 1x TAE buffer (Bio-Rad 1610773), containing 1x SYBR Safe DNA Gel Stain (Thermo Fisher S33102) on a Bio-Rad PowerPac HC electrophoresis power supply at 120V, 40 min. DNA inserts were incorporated into plasmids using In-Fusion HD Enzyme premix according to the manufacturer's protocol (Takara) and transformed into Stellar Competent Cells (Takara), an E. coli HST08 strain. DNA sequences are reported in the supplementary methods.

\section{Resuspending cells prior to flow cytometry or ADCC (Procedure \#1)}

In all cases, breast cancer cells were split/processed 2-3 days prior to an assay, plated on a T75 culture flask (Thermo Fisher), and allowed to grow to $60-90 \%$ confluence. To lift cells for an assay, cells were first washed $1 \mathrm{x}$ with room temperature PBS $-\mathrm{Ca}-\mathrm{Mg}$, $(10 \mathrm{~mL})$, then $7 \mathrm{~mL}$ cell dissociation buffer pre-warmed to $37{ }^{\circ} \mathrm{C}$ was added and cells were incubated at $37{ }^{\circ} \mathrm{C}$ with $5 \% \mathrm{CO}_{2}$ until just lifted off the plate $(5-20 \mathrm{~min})$. Cell flasks were rinsed vigorously with $7 \mathrm{~mL}$ normal growth media, transferred to $15 \mathrm{~mL}$ falcon tubes, and pelleted by centrifugation at $300 \times g$ for $5 \mathrm{~min}$. Cells were resuspended in 2-5 $\mathrm{mL}$ assay media, counted with a Countess II FL Automated Cell Counter (Thermo Fisher Scientific), and then diluted in assay media to desired concentration for assay.

\section{General procedure for desialylation of cells (Procedure \#2)}


Cells were lifted as described in Procedure \#1 and diluted to $1 \times 10^{6}$ cells $/ \mathrm{mL}$ in normal growth media. $200 \mu \mathrm{L}$ of cells were added to $\mathrm{V}$ bottom 96-well plates (for mixed cell assays, $100 \mu \mathrm{L}$ MDA-MB-468 and $100 \mu \mathrm{L}$ of SK-BR-3 were added to make $200 \mu \mathrm{L}$ final well volumes). Individual wells were treated with various concentrations of sialidase, conjugates, or equivalent volume PBS. Cells were incubated with constructs for $1 \mathrm{~h}$ at $37^{\circ} \mathrm{C}, 5 \% \mathrm{CO}_{2}$. Following this, cells were pelleted in the plates by centrifugation at 300 $x g$ for 5 min. Supernatant was removed and replaced with $200 \mu \mathrm{L}$ PBS; this was repeated a total of three times to complete washing of the cells prior to staining.

\section{SNA staining procedure}

Following desialylation at various sialidase/T-Sia concentrations as described in Procedure 2, cells were resuspended in PBS+0.5\%BSA containing Alexa Fluor ${ }^{\circledR} 647$ anti-human Her2 antibody and (FITC)-labeled Sambucus nigra lectin and incubated for 30 min at $4{ }^{\circ} \mathrm{C}$ in the dark. Cells were then washed $3 x$ in PBS $+0.5 \%$ BSA, resuspended in PBS $+0.5 \%$ BSA, and analyzed by flow cytometry on a BD Accuri C6 Plus Flow Cytometer (BD Biosciences). Flow cytometry data was analyzed using FlowJo v. 10.0 software (Tree Star) and gated to distinguish HER2 ${ }^{+}$and HER2 ${ }^{-}$cells as well as to quantify $\mathrm{SNA}^{+}$and $\mathrm{SNA}^{-}$cells. For the flow plot in Fig. 2b, >16,000 cells are shown for each antibody treatment condition. For the analysis in Fig. 2a,c, \& Supplementary Fig. $5>5,000$ cells are reported for each treatment.

\section{Procedure for Siglec-Fc staining of cancer cells}

Breast cancer cell lines were treated as described in Procedure \#1, followed by treatment with $2 \mu \mathrm{M} \mathrm{ST}$ sialidase for $1 \mathrm{~h}$ (Procedure \#2). After washing, cells were resuspended in PBS $+0.5 \%$ BSA containing Siglec-7, -9 , or F-Fc that had pre-incubated for 30 min at $4{ }^{\circ} \mathrm{C}$ with Alexa Fluor ${ }^{\circledR} 488$ AffiniPure Goat Anti-Human IgG, or Siglec-E$\mathrm{mFc}$ pre-incubated for $30 \mathrm{~min}$ at $4{ }^{\circ} \mathrm{C}$ with Alexa Fluor® 647 AffiniPure Goat Anti-Mouse IgG. Stain was incubated with the cells for $30 \mathrm{~min}$ at $4{ }^{\circ} \mathrm{C}$, followed by $3 x$ washes and resuspension in PBS+0.5\% BSA and analysis by flow cytometry (BD Accuri C6 Plus or LSR II). Gating was performed using FlowJo software to eliminate debris (FSC/SSC) 
and analyze single cells (FSC-A/FSC-H), at least 2,000 cells are reported for each replicate and treatment, except when otherwise noted as in Supplementary Fig. 17.

\section{Determining $\mathrm{K}_{\mathrm{D}}$ of T-Sia 2}

HER2 ${ }^{+}$HCC-1954 cells were lifted (Procedure \#1), resuspended to $1 \times 10^{6} \mathrm{cells} / \mathrm{mL}$ in PBS $+0.5 \%$ BSA, and $180 \mu \mathrm{L}$ were distributed into wells of a 96-well V-bottom plate (Corning). Various concentrations $(20 \mathrm{nM}-0.01 \mathrm{nM})$ of trastuzumab or T-Sia 2 were added to the cells in equal volumes and incubated with cells for $30 \mathrm{~min}, 4^{\circ} \mathrm{C}$. Following this, cells were washed $3 \times$ in PBS $+0.5 \%$ BSA, pelleting by centrifugation at $300 \times g$ for 5 min between washes. Cells were resuspended in Alexa Fluor 488 AffiniPure Goat Anti-Human IgG in PBS $+0.5 \%$ BSA for 15 min at $4{ }^{\circ} \mathrm{C}$. Cells were further washed $2 x$ and resuspended in PBS $+0.5 \%$ BSA and fluorescence was analyzed by flow cytometry (BD Accuri C6 Plus). Gating was performed using FlowJo v. 10.0 software (Tree Star) to eliminate debris and isolate single cells. MFI (median fluorescence intensity) of the cell populations were subtracted from control cells treated with secondary antibody only, and normalized to the maximum MFI population from each experimental replicate. Values were fit to a one site - total binding curve using GraphPad Prism 6, which calculated the $K_{D}$ values as the antibody concentration needed to achieve a halfmaximum binding.

\section{General procedure for activity assay of sialidases using 4-MUNANA.}

Sialidases were diluted (10 pM -1 $\mu \mathrm{M})$ in DPBS containing $\mathrm{Ca}^{2+}$ and $\mathrm{Mg}^{2+}$ in a 96-well clear bottom black microplate (Corning 3904). Immediately before beginning the read,

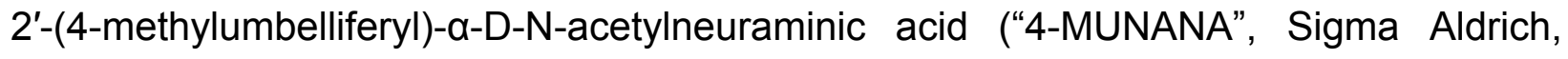
M8639) was added to the sialidase solutions solution to a $1 \mathrm{mM}$ final concentration, with a final solution volume of $100 \mu \mathrm{L}$. Coumarin release was measured by fluorescence (excitation $365 \mathrm{~nm}$; emission $450 \mathrm{~nm}$ ) in the SpectraMax i3x plate reader at $37^{\circ} \mathrm{C}$. Background fluorescence was subtracted from control wells lacking enzyme, and fluorescence was compared to a standard curve of 4-methylumbelliferone $(0-150 \mu \mathrm{M})$ (Sigma Aldrich, M1381) to calculate the amount of hydrolyzed substrate. Specific 
activity (reported $\mu \mathrm{mol}$ substrate converted $\times \mathrm{min}^{-1}$ per $\mathrm{mg}$ enzyme) was calculated from the rate of hydrolysis in the initial linear range and $\mathrm{mg}$ of enzyme.

\section{General procedure for an LDH ADCC assay}

After lifting the cells (Procedure \#1, above) Cells were resuspended in 2-5 mL phenolred-free RPMI $+1 \%$ heat-inactivated FBS, counted with a Countess II FL Automated Cell Counter (Thermo Fisher Scientific), and then diluted in media to desired concentration. $1 \times 10^{4}$ cells were plated in 96-well V-bottom plates (Corning), and trastuzumab, sialidase, conjugates, or PBS were added to a final volume of $100 \mu \mathrm{L}$ and treatments were pre-incubated with target breast cancer cells for $30 \mathrm{~min}$. Next, $100 \mu \mathrm{L}$ of effector cells in phenol-red free RPMI $+1 \%$ heat-inactivated FBS were added at E/T ratios ranging from 0-15 for the various assays. The assay plate was incubated at 37 ${ }^{\circ} \mathrm{C}+5 \% \mathrm{CO}_{2}$ for $4-8 \mathrm{~h}$. Cells were pelleted by centrifugation at $500 \times \mathrm{g}, 5 \mathrm{~min}$, and 50 $\mu \mathrm{L}$ of supernatant was transferred to a 96 well flat-bottom microplate to perform the LDH Cytotoxicity Assay Kit (Pierce, 88953) according to the manufacturers protocol. Absorbance was measured with the SpectraMax i3x plate reader at $490 \mathrm{~nm}$ and 680 $\mathrm{nm}$, final cytotoxicity was calculated according to the assay kit manufacturer's protocol.

\section{NK cell-mediated ADCC by flow cytometry against EMT6 target cells}

Cells were lifted according to Procedure \#1. Cells were resuspended in serum-free media containing $5 \mu \mathrm{M}$ CellTracker ${ }^{\mathrm{TM}}$ Green CMFDA Dye (Thermo Fisher Scientific, $\mathrm{C} 7025$ ) and incubated at $37{ }^{\circ} \mathrm{C}$ for $30 \mathrm{~min}$ in $5 \% \mathrm{CO}_{2}$. Cells were recollected by centrifugation at $300 \times g$ for $5 \mathrm{~min}$ and resuspended in 2-5 mL normal growth media, counted with a Countess II FL Automated Cell Counter (Thermo Fisher Scientific), and then diluted in media to desired concentration. Then, $2 \times 10^{4}$ cells $/ \mathrm{mL}$ cells were pretreated with trastuzumab, PBS, or T-Sia 2 for $30 \mathrm{~min}$ at a final volume of $100 \mu \mathrm{L}$ and human NK cells (without IL-2 pre-treatment) were added at an effector:target ratio of 3:1. After 4 h, SYTOX ${ }^{\text {TM }}$ Red Dead Cell Stain (Thermo Fisher Scientific, S34859) was added at $5 \mathrm{nM}$ final concentration to the cell mixture, which was analyzed on the BD Accuri C6+ flow cytometer (BD Biosciences). Using FlowJo v. 10.0 software (Tree Star), cells positive for CellTracker green in the FL1 channel were selected and gated 
for live/dead by the red FL4 channel. Data are presented with PBS-treated cells subtracted from the trastuzumab and T-Sia 2 treated mixtures (Fig. 4A)

\section{Cloning and expression of proteins (antibodies, FGE, and sialidases)}

Cloning, sequences, and expression of proteins are described in the supplemental methods.

\section{Representative Procedure for Antibody cysteine $\rightarrow$ fGly conversion}

tbFGE in TEAM buffer (25 mM triethanolamine, $50 \mathrm{mM} \mathrm{NaCl}, \mathrm{pH}$ 9) $(101.4 \mu \mathrm{L}$, final 0.9 $\mu \mathrm{M}, 0.1$ equiv.) was added to Motavizumab in TEAM buffer $(3.7 \mathrm{~mL}$, final $9 \mu \mathrm{M}, 1$ equiv.) and $\mathrm{CuSO}_{4}$ (5 mM stock in $\mathrm{MQ}$ water) was immediately added to $5 \mu \mathrm{M}$ final concentration. 2-Mercaptoethanol was added for a final concentration of $2 \mathrm{mM}$. Conversion occurred at $37{ }^{\circ} \mathrm{C}, 400 \mathrm{rpm}$ for $16 \mathrm{~h}$ and was analyzed by ESI-TOF mass spectrometry. Detection of and quantification of fGly conversion is difficult because the shift is small $(-17 \mathrm{Da})$ and can exist as a hydrate very close to the original antibody molecular weight $(-1 \mathrm{Da})$. Antibodies were re-purified and concentrated by protein $\mathrm{A}$ chromatography as described above and proceeded onto the next step.

\section{Representative procedure for HIPS-azide addition to antibody}

Trastuzumab fGly was buffer exchanged by PD-10 column (GE Life Sciences, 17085101 ) into $50 \mathrm{mM}$ sodium citrate and $50 \mathrm{mM} \mathrm{NaCl}, \mathrm{pH}$ 5.5. A HIPS-azide aliquoted solid stock was removed from $-80^{\circ} \mathrm{C}$, dissolved in DMSO to $36 \mathrm{mM}$, and added (11.987 $\mu \mathrm{mol}, 20$ equiv., $333 \mu \mathrm{L})$ to trastuzumab fGly in citrate buffer $(600.6 \mathrm{nmol}, 1$ equiv., $7.947 \mathrm{~mL}$ ). Mixture was shaken at $230 \mathrm{rpm}, 37^{\circ} \mathrm{C}$ overnight, followed by PD-10 buffer exchange into PBS. Only HIPS converted trastuzumab was detected, with a $96 \%$ recovery of protein. Methods for copper-click labeling of fluorophore onto HIPS-azide labeled antibodies and subsequent stability assays are further described in the Supplementary methods.

\section{Procedure for $\alpha$-chloroacetamide labeling of ST sialidase}


ST sialidase $(1.04 \mu \mathrm{mol}, 1$ equiv., $16 \mathrm{~mL})$ in $50 \mathrm{mM}$ ammonium bicarbonate buffer $\mathrm{pH}$ 8.3 was incubated in the dark with TCEP ( $2 \mathrm{mM}$ final concentration, $66 \mu \mathrm{L}$ addition), while shaking at $300 \mathrm{rpm}$ for $10 \mathrm{~min}$. a-chloroacetamide-DBCO was dissolved into DMSO to $50 \mathrm{mM}$ and added to the ST sialidase solution (20.88 $\mu \mathrm{mol}, 20$ equiv., 417.6 $\mu \mathrm{L}$ ), and the mixture was shaken in the dark at $300 \mathrm{rpm}$ overnight at room temperature in a $50 \mathrm{~mL}$ falcon tube. Reaction completion was determined by desalting an aliquot (Zeba spin desalting columns, $7 \mathrm{k}$ MWCO, Thermo), and performing UV-vis measurements at $280 \mathrm{~nm}$ and $309 \mathrm{~nm}$ to check for \%DBCO conjugated. Once fully conjugated, purification was performed using a HiLoad 26/600 Superdex 75 pg on the ÄKTA pure chromatography system in PBS at $4{ }^{\circ} \mathrm{C}$, followed by $0.2 \mu \mathrm{m}$ syringe filtration to avoid contamination.

\section{Maleimide-PEG4-DBCO labeling of ST sialidase}

To ST sialidase ( $618 \mathrm{nmol}, 30 \mathrm{~mL}, 1$ equiv.) in PBS, TCEP was added ( $30 \mu \mathrm{mol}, 60 \mu \mathrm{L})$ (Fisher Scientific, T2556) and the mixture was rotated at $4{ }^{\circ} \mathrm{C}$ for 30 min protected from

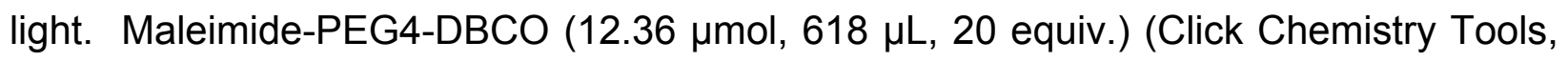
$\mathrm{A} 108 \mathrm{P}$ ) in DMSO was added and the reaction was mixed at $600 \mathrm{rpm}$ for 2.5 days at 4 ${ }^{\circ} \mathrm{C}$. Purification was performed using a HiLoad $26 / 600$ Superdex 75 pg on the ÄKTA pure chromatography system in PBS.

\section{Representative synthesis of antibody-sialidase conjugate}

T-FcX-HIPS-azide (63.3 nmol, 1 equiv. $11.9 \mathrm{~mL}$ ) and ST sialidase ( $316.9 \mathrm{nmol}, 5$ equiv., $16 \mathrm{~mL}$ ), both in PBS buffer, were mixed together and concentrated to $\sim 25 \mathrm{mg} / \mathrm{mL}$ using 10,000 MWCO Amicon spin filters. The final mixture $(\sim 0.92 \mathrm{~mL})$ was incubated at $25^{\circ} \mathrm{C}$ in the dark with shaking at $500 \mathrm{rpm}$ for 3 days. The reaction was monitored by SDSPAGE and purified by size exclusion chromatography using a Superdex 200 increase $10 / 300$ column on the ÄKTA pure chromatography system in PBS (GE Healthcare Life Sciences) to remove unconjugated and aggregated protein, followed by protein $A$ chromatography to re-concentrate and further purify the antibody sialidase conjugates. Final conjugates were buffer exchanged to PBS buffer with PD-10 columns and $0.2 \mu \mathrm{m}$ syringe filtered for sterility. 


\section{Endotoxin detection}

The HEK-Blue LPS Detection Kit (Invivogen, rep-Ips2) was used according to the manufacturer's protocol. Briefly, serial dilutions of antibody-enzyme conjugates were incubated with HEK-Blue cells both alone and spiked with $0.1 \mathrm{EU} / \mathrm{mL}$ Endotoxin standard to verify that no inhibition occurred. After $24 \mathrm{~h}$, an aliquot of the cell media was incubated with QUANTI-Blue reagent (Invivogen) and absorbance was read on a SpectraMax i3x Multi-Mode microplate reader and compared to a standard curve. For all in vitro cell experiments and mouse experiments, endotoxin of the antibody-enzyme conjugates was determined to be $<1 \mathrm{EU} / \mathrm{mg}$.

\section{RP-HPLC}

Antibody conjugates (1 $\mathrm{mg} / \mathrm{mL}$ ) were buffer exchanged into $100 \mathrm{mM}$ ammonium bicarbonate $+8 \mathrm{M}$ urea in water $(\mathrm{pH}$ 8.3). Stock DTT was added to $5 \mathrm{mM}$ final concentration and antibody was heated to $56{ }^{\circ} \mathrm{C}$, with shaking at $700 \mathrm{rpm}$ for $25-45 \mathrm{~min}$ in an Eppendorf thermomixer. The sample was allowed to cool, spun down briefly in tabletop centrifuge, and $14 \mathrm{mM}$ iodoacetamide was added from a freshly prepared 500 $\mathrm{mM}$ stock in water; sample was protected from light and incubated at $25^{\circ} \mathrm{C}$ with shaking at $700 \mathrm{rpm}$ for $30 \mathrm{~min}$. The reaction was quenched by addition of another $5 \mathrm{mM}$ DTT and incubated again in the dark, at RT, $700 \mathrm{rpm}, 15 \mathrm{~min}$. Reversed-phase high performance liquid chromatography was performed on an 1100/1200 series instrument (Agilent Technologies) connected in-line to a UV-vis spectrophotometer. A total of 10 $\mu \mathrm{g}$ protein was injected onto a Zorbax $3.5 \mu \mathrm{m} 300 \mathrm{SB} 300 \AA$ C8 2.1 x $50 \mathrm{~mm}$ column (Agilent). RP-HPLC was performed at $0.9 \mathrm{~mL} / \mathrm{min}$ at $60{ }^{\circ} \mathrm{C}$ using $0.1 \%$ trifluoroacetic acid (TFA) in water (mobile phase $A$ ) and $0.1 \%$ TFA in acetonitrile (mobile phase $B$, MPB). The 28 minute method consisted of a $4 \mathrm{~min}$ isocratic hold at $28 \% \mathrm{MPB}$, a linear gradient for $6 \mathrm{~min}$ to $34 \% \mathrm{MBP}$, an isocratic hold at 34\% MBP for $1.5 \mathrm{~min}$ to increase the separation between $\mathrm{HC}$ and $\mathrm{HC}-\mathrm{Sia}$, and a 6.5 min linear gradient to $42 \% \mathrm{MBP}$, followed by a 5 min wash using $100 \% \mathrm{MBP}$, and a $5 \mathrm{~min}$ re-equilibration at $28 \% \mathrm{MBP}$. Antibody/Enzyme ratio (EAR) was calculated by integrating the area-under-the-curve of the light chain and heavy chain peaks and calculating molar ratios of the antibody 
chains using the protein extinction coefficients at $\mathrm{A}_{280}$. When $\mathrm{HC}$ and $\mathrm{HC}$-Sia were not perfectly separated, as in the case of motavizumab, the equation of $[\mathrm{LC}]=[\mathrm{HC}]+[\mathrm{HC}$ Sia] and the known extinction coefficients of each of the three chains was used to determine the EAR.

\section{Mass spectrometry of full-length proteins}

Protein samples $(30 \mu \mathrm{L}, \sim 10 \mu \mathrm{M})$ in PBS or $50 \mathrm{mM}$ Ammonium bicarbonate buffer were treated with $1 \mu \mathrm{L}$ PNGaseF (New England Biolabs, P0704S) overnight at $37{ }^{\circ} \mathrm{C}$. Following this, the samples were analyzed by ESI-LC/MS on an Agilent $1260 \mathrm{HPLC}$ and Bruker MicroTOF-Q II time-of-flight mass spectrometer. The column was a Waters BioResolve RP mAb Polyphenyl 450A 2.7u $100 \times 2.1 \mathrm{~mm}$ maintained at $50^{\circ} \mathrm{C}$; the flow rate was $0.3 \mathrm{~mL} / \mathrm{min}$, and the injection volume was $5 \mu \mathrm{L}$. Solvent $A$ was $0.095 \%$ formic acid and $0.05 \%$ TFA in water, solvent $B$ was $0.1 \%$ formic acid in acetonitrile. The gradient began with $5 \% \mathrm{~B}$ held for $1.5 \mathrm{~min}$ then ramped to $35 \% \mathrm{~B}$ at $2 \mathrm{~min}, 46 \%$ B at 10 min, and $95 \%$ B at 11 min held for 1 minute. Data was collected in full scan MS mode with a mass range of 400-4000 Da with a Collision RF setting of $800 \mathrm{~V}$. The protocol for trypsin digest and mass spectrometry of digested proteins is detailed in the Supplemental methods

\section{T-Sia 2 treatment of EMT6 tumors in mice}

All mouse experiments were approved by the local Ethical Committee (Basel Stadt). BALB/c mice were obtained from Janvier Labs (France) and bred in-house at the University Hospital Basel, Switzerland. Animals were housed under specific pathogenfree conditions.

For tumor growth experiments, 7-9 week old females were used. EMT6-HER2 cells were injected into the right mammary gland of female BALB/c mice $\left(1 \times 10^{6}\right.$ cells in $40 \mu \mathrm{L}$ of PBS). Tumor size and health score, as well as weight, were measured and monitored three times weekly. Perpendicular tumor diameters were measured by caliper and tumor volume calculated according to the following formula: tumor volume $\left(\mathrm{mm}^{3}\right)=$ $\left(d^{2 *} D\right) / 2$, where $d$ and $D$ are the shortest and longest diameters of the tumor (in 
millimeters), respectively. Mice were sacrificed once tumor size reached $1000-500 \mathrm{~mm}^{3}$. Animals developing ulcerated tumors were removed from the further analysis. All treatments were given intraperitoneally. A total of four doses were administered every second to third day once the tumor size reached approx. $100 \mathrm{~mm}^{3}$.

\section{Sig- $\mathrm{E}^{-/-}$mouse experiments}

Sig- $\mathrm{E}^{-/}$C57BL/6 mice were provided by Paul Crocker, College of Life Sciences, University of Dundee, Dundee, UK ${ }^{57}$ and bred in-house at the University Hospital Basel, Switzerland. Animals were housed under specific pathogen-free conditions. Briefly, 500,000 B16D5 cells expressing the HER2 protein were resuspended in PBS and injected subcutaneously into the flanks of C57BL/6 mice (wt or Sig- $\mathrm{E}^{-/}$). After 7 days, mice were injected IP with $10 \mathrm{mg} / \mathrm{kg} \mathrm{T-Sia} \mathrm{2,} \mathrm{T-Sia-LOF,} \mathrm{or} \mathrm{PBS} \mathrm{4x} \mathrm{over} 2$ weeks. Tumor size was measured as described above every 2-4 days until tumors reached $1500 \mathrm{~mm}^{3}$.

\section{Leukocyte analysis}

For analysis of tumor-infiltrating immune cells, resected tumors were mechanically dissociated and digested with a mixture of Accutase, collagenase IV, hyaluronidase and DNAse type IV. Samples were filtered through a $70 \mu \mathrm{m}$ mesh and tumor-infiltrating lymphocytes were enriched by density centrifugation using Histopaque-1119 (Sigma). Samples were frozen (90\% FCS, 10\% DMSO) and stored in liquid nitrogen until further analysis.

Multicolor flow cytometry was performed on single cell suspensions. Samples were incubated with fixable live/dead dye and Fc receptor block followed by staining with primary antibodies. Stained samples were fixed with IC fixation buffer (eBioscience) until time of analysis. For intracellular staining, samples were permeabilized after fixation. All tumor samples were analyzed by flow cytometry using a Fortessa LSR II flow cytometer (BD Biosciences, USA) and cells analyzed after serial duplet exclusion and live/dead discrimination using FlowJo v. 10.0 software (Tree Star).

\section{Lectin staining of mouse tumor digests}


Lectin staining was performed using biotinylated PNA, SNA, MALII, and ConA obtained from Vector Laboratories (USA). Single cell suspensions of tumor digests were incubated with lectins at $10 \mu \mathrm{g} / \mathrm{ml}$ and detected in a second step using Streptavidin-PECy7 or Streptavidin-AF647, both for 20 minutes at $4{ }^{\circ} \mathrm{C}$. Sialylation was assessed by flow cytometry using a CytoFLEX (Beckman Coulter) cytometer or an LSR II and quantified after live/dead and duplet exclusion using the geometric mean of PNA staining. ConA was used as a sialic acid-independent control.

\section{T-Sia 2 imaging in mice}

T-Sia 2 was labeled with $3 x$ excess IRDye $® 800 \mathrm{CW}$ NHS ester at $\mathrm{pH} 8.3$ in PBS for $1 \mathrm{~h}$. After buffer exchanging to PBS, fluorophore labeling was quantified by nanodrop UV measurements at $280 \mathrm{~nm}$ and $774 \mathrm{~nm}$. T-Sia was found to have 1.7 fluorescent molecules for every T-Sia 2 conjugate, which was then diluted to a 1:1 fluorophore:conjugate ratio using unlabeled T-Sia 2. Balb/c mice were then injected subcutaneously with $1 \times 10^{6}$ cells in $100 \mu \mathrm{L}$ PBS, after 5 days, mice were injected IP with fluorophore-labeled T-Sia 2 . Live mice were anesthetized with isoflurane and imaged at 2 and 4 days on an IVIS Lumina instrument (ex. $700 \mathrm{~nm}$, em: $790 \mathrm{~nm}$, 2s exposure time), then sacrificed and organs and tumors were imaged ex vivo on the IVIS Lumina. Tumors were then resuspended and stained for lectin binding as described above.

\section{Blood counts}

Blood was collected from the tail vein of PBS, trastuzumab and T-Sia 2 treated mice (Fig. 5) into EDTA-coated Microtainers (BD Biosciences) 48h after the first dose of treatment. Generally, $40-60 \mu \mathrm{L}$ of blood were diluted to a total volume of $240 \mu \mathrm{L}$ using $0.9 \% \mathrm{NaCl}$. Complete blood counts were measured on the ADVIA120 Hematology Analyzer using the Multispecies Version 5.9.0-MS software (Bayer) and adjusted to the respective dilution factor. 


\section{References}

1. Schadendorf, D. et al. Pooled analysis of long-term survival data from phase II and phase III trials of ipilimumab in unresectable or metastatic melanoma. J. Clin. Oncol. 33, 1889-1894 (2015).

2. Ribas, A. et al. Association of pembrolizumab with tumor response and survival among patients with advanced melanoma. J. Am. Med. Assoc. 315, 1600-1609 (2016).

3. Sharma, P., Hu-Lieskovan, S., Wargo, J. A. \& Ribas, A. Primary, adaptive, and acquired resistance to cancer immunotherapy. Cell 168, 707-723 (2017).

4. Du, W. et al. TIM-3 as a target for cancer immunotherapy and mechanisms of action. Int. J. Mol. Sci. 18, 645 (2017).

5. Woo, S.-R. et al. Immune inhibitory molecules LAG-3 and PD-1 synergistically regulate T-cell function to promote tumoral immune escape. Cancer Res. $\mathbf{7 2}$, 917-927 (2012).

6. Emens, L. A. et al. CPI-444, an oral adenosine A2A receptor (A2AR) antagonist, demonstrates clinical activity in patients with advanced solid tumors. in AACR (2017).

7. Advani, R. et al. CD47 blockade by Hu5F9-G4 and rituximab in non-Hodgkin's lymphoma. N. Engl. J. Med. 379, 1711-1721 (2018).

8. Thompson, J. A. et al. A phase 1 dose-escalation trial of intratumoral TTI-621, a novel immune checkpoint inhibitor targeting CD47, in subjects with relapsed or refractory percutaneously-accessible solid tumors and mycosis fungoides. J. Clin. Oncol. 35, TPS3101 (2017).

9. Bilir, C. \& Sarisozen, C. Indoleamine 2,3-dioxygenase (IDO): Only an enzyme or a checkpoint controller? J. Oncol. Sci. 3, 52-56 (2017).

10. Ishizuka, J. J. et al. Loss of ADAR1 in tumours overcomes resistance to immune checkpoint blockade. Nature 565, 43-48 (2019).

11. Marin-Acevedo, J. A. et al. Next generation of immune checkpoint therapy in cancer: new developments and challenges. J. Hematol. Oncol. 11, 39 (2018).

12. $\mathrm{Li}, \mathrm{C}$--W. et al. Eradication of triple-negative breast cancer cells by targeting glycosylated PD-L1. Cancer Cell 33, 187-201 (2018).

13. Boligan, K. F., Mesa, C., Fernandez, L. E. \& von Gunten, S. Cancer intelligence acquired $(\mathrm{CIA})$ : tumor glycosylation and sialylation codes dismantling antitumor defense. Cell. Mol. Life Sci. 72, 1231-1248 (2015).

14. Varki, A., Kannagi, R., Toole, B. \& Stanley, P. Glycosylation changes in cancer. in Essentials of Glycobiology (Cold Spring Harbor Laboratory Press, 2017).

15. Cohen, M. et al. Sialylation of 3-methylcholanthrene-induced fibrosarcoma determines antitumor immune responses during immunoediting. J. Immunol. 185, 5869-5878 (2010).

16. Perdicchio, M. et al. Tumor sialylation impedes $T$ cell mediated anti-tumor responses while promoting tumor associated-regulatory T cells. Oncotarget 7 , 8771-8782 (2016).

17. Stanczak, M. A. et al. Self-associated molecular patterns mediate cancer immune evasion by engaging Siglecs on T cells. J. Clin. Invest. 128, 4912-4923 (2018).

18. Büll, C., Stoel, M. A., den Brok, M. H. \& Adema, G. J. Sialic acids sweeten a 
tumor's life. Cancer Res. 74, 3199-3204 (2014).

19. Varki, A. \& Gagneux, P. Multifarious roles of sialic acids in immunity. Ann. N. Y. Acad. Sci. 1253, 16-36 (2012).

20. Swindall, A. F. \& Bellis, S. L. Sialylation of the Fas death receptor by ST6Gal-I provides protection against Fas-mediated apoptosis in colon carcinoma cells. J. Biol. Chem. 286, 22982-22990 (2011).

21. Lübbers, J., Rodríguez, E. \& van Kooyk, Y. Modulation of Immune Tolerance via Siglec-Sialic Acid Interactions. Front. Immunol. 9, 2807 (2018).

22. Macauley, M. S., Crocker, P. R. \& Paulson, J. C. Siglec-mediated regulation of immune cell function in disease. Nat. Rev. Immunol. 14, 653-666 (2014).

23. Paulson, J. C., Macauley, M. S. \& Kawasaki, N. Siglecs as sensors of self in innate and adaptive immune responses. Ann. N. Y. Acad. Sci. 1253, 37-48 (2012).

24. Riley, J. L. PD-1 signaling in primary T cells. Immunol. Rev. 229, 114-125 (2009).

25. Chemnitz, J. M., Parry, R. V, Nichols, K. E., June, C. H. \& Riley, J. L. SHP-1 and SHP-2 associate with immunoreceptor tyrosine-based switch motif of programmed death 1 upon primary human T cell stimulation, but only receptor ligation prevents T cell activation. J. Immunol. 173, 945-954 (2004).

26. Watson, H. A., Wehenkel, S., Matthews, J. \& Ager, A. SHP-1: the next checkpoint target for cancer immunotherapy? Biochem. Soc. Trans. 44, 356-362 (2016).

27. Läubli, H. et al. Engagement of myelomonocytic Siglecs by tumor-associated ligands modulates the innate immune response to cancer. Proc. Natl. Acad. Sci. U. S. A. 111, 14211-14216 (2014).

28. Hudak, J. E., Canham, S. M. \& Bertozzi, C. R. Glycocalyx engineering reveals a Siglec-based mechanism for NK cell immunoevasion. Nat. Chem. Biol. 10, 69-75 (2014).

29. Büll, C. et al. Sialic acid blockade suppresses tumor growth by enhancing T-cellmediated tumor immunity. Cancer Res. 78, 3574-3588 (2018).

30. Xiao, H., Woods, E. C., Vukojicic, P. \& Bertozzi, C. R. Precision glycocalyx editing as a strategy for cancer immunotherapy. Proc. Natl. Acad. Sci. U. S. A. 113, 10304-10309 (2016).

31. Thobhani, S., Ember, B., Siriwardena, A. \& Boons, G.-J. Multivalency and the mode of action of bacterial sialidases. J. Am. Chem. Soc. 125, 7154-7155 (2003).

32. Watson, J. N. et al. Use of conformationally restricted pyridinium-D-Nacetylneuraminides to probe specificity in bacterial and viral sialidases. Biochem. Cell Biol 83, 115-122 (2005).

33. Minami, A. et al. Catalytic preference of Salmonella typhimurium LT2 sialidase for $\mathrm{N}$-acetylneuraminic acid residues over $\mathrm{N}$-glycolylneuraminic acid residues. FEBS Open Bio 3, 231-236 (2013).

34. Shepard, H. M., Jin, P., Slamon, D. J., Pirot, Z. \& Maneval, D. C. Herceptin. in Therapeutic Antibodies 183-219 (Springer, Berlin, Heidelberg, 2008).

35. Agarwal, P., van der Weijden, J., Sletten, E. M., Rabuka, D. \& Bertozzi, C. R. A Pictet-Spengler ligation for protein chemical modification. Proc. Natl. Acad. Sci. U. S. A. 110, 46-51 (2013).

36. Agarwal, P. et al. Hydrazino-Pictet-Spengler ligation as a biocompatible method for the generation of stable protein conjugates. Bioconjug. Chem. 24, 846-851 
(2013).

37. Barfield, R. M. \& Rabuka, D. Leveraging Formylglycine-Generating Enzyme for Production of Site-Specifically Modified Bioconjugates. in Noncanonical Amino Acids 3-16 (Humana Press, New York, NY, 2018).

38. Drake, P. M. et al. Aldehyde tag coupled with HIPS chemistry enables the production of ADCs conjugated site-specifically to different antibody regions with distinct in vivo efficacy and PK outcomes. Bioconjug. Chem. 25, 1331-1341 (2014).

39. Maclaren, A., Levin, N., Lowman, H. \& Trikha, M. Trph-222, a novel anti-CD22 antibody drug conjugate (ADC), has signficant anti-tumor activity in $\mathrm{NHL}$ xenografts and is well tolerated in non-human primates. Blood 130, 4105 (2017).

40. Study of TRPH-222 in patients with relapsed and/or refractory B-cell lymphoma. Clinicaltrials.gov (2018). ClinicalTrials.gov Identifier: NCT03682796

41. Baskin, J. M. \& Bertozzi, C. R. Copper-free click chemistry: bioorthogonal reagents for tagging azides. Aldrichimica Acta 43, 15-23 (2010).

42. Rockwell, S. C., Kallman, R. F. \& Fajardo, L. F. Characteristics of a serially transplanted mouse mammary tumor and its tissue-culture-adapted derivative. J. Natl. Cancer Inst. 49, 735-749 (1972).

43. D'Amico, L. et al. A novel anti-HER2 anthracycline-based antibody-drug conjugate induces adaptive anti-tumor immunity and potentiates PD-1 blockade in breast cancer. J. Immunother. Cancer 7, 16 (2019).

44. Wu, H. et al. Development of motavizumab, an ultra-potent antibody for the prevention of respiratory syncytial virus infection in the upper and lower respiratory tract. J. Mol. Biol. 368, 652-665 (2007).

45. Kelly, R. L. et al. High throughput cross-interaction measures for human IgG1 antibodies correlate with clearance rates in mice. MAbs 7, 770-777 (2015).

46. Armour, K. L., Clark, M. R., Hadley, A. G. \& Williamson, L. M. Recombinant human IgG molecules lacking Fcy receptor I binding and monocyte triggering activities. Eur. J. Immunol. 29, 2613-2624 (1999).

47. Shields, R. L. et al. High resolution mapping of the binding site on human IgG1 for FcyRI, FcyRII, FcyRIII, and FcRn and design of IgG1 variants with improved binding to the FcyR. J. Biol. Chem. 276, 6591-6604 (2001).

48. $\mathrm{Li}, \mathrm{J}$. et al. Desialylation is a mechanism of Fc-independent platelet clearance and a therapeutic target in immune thrombocytopenia. Nat. Commun. 6, 7737 (2015).

49. Tribulatti, M. V., Mucci, J., van Rooijen, N., Leguizamón, M. S. \& Campetella, O. The trans-sialidase from Trypanosoma cruzi induces thrombocytopenia during acute Chagas' disease by reducing the platelet sialic acid contents. Infect. Immun. 73, 201-207 (2005).

50. Assi, H., Ibrahimi, S., Machiorlatti, M., Vesely, S. K. \& Asch, A. S. Thrombocytopenia is a biomarker for response in patients treated with anti PD1/PDL-1 therapy. Blood 132, 1138 (2018).

51. Kurozumi, S. et al. Prognostic utility of tumor-infiltrating lymphocytes in residual tumor after neoadjuvant chemotherapy with trastuzumab for HER2-positive breast cancer. Sci. Rep. 9, 1583 (2019).

52. Dieci, M. V. et al. Prognostic value of tumor-infiltrating lymphocytes on residual disease after primary chemotherapy for triple-negative breast cancer: a 
retrospective multicenter study. Ann. Oncol. 25, 611-618 (2014).

53. Lança, T. \& Silva-Santos, B. The split nature of tumor-infiltrating leukocytes: Implications for cancer surveillance and immunotherapy. Oncoimmunology 1, 717-725 (2012).

54. Laoui, D. et al. Tumor-associated macrophages in breast cancer: distinct subsets, distinct functions. Int. J. Dev. Biol. 55, 861-867 (2011).

55. Souza-Fonseca-Guimaraes, F., Cursons, J. \& Huntington, N. D. The emergence of natural killer cells as a major target in cancer immunotherapy. Trends Immunol. 40, 142-158 (2019).

56. Fogel, L. A., Sun, M. M., Geurs, T. L., Carayannopoulos, L. N. \& French, A. R. Markers of nonselective and specific NK cell activation. J. Immunol. 190, 62696276 (2013).

57. McMillan, S. J. et al. Siglec-E is a negative regulator of acute pulmonary neutrophil inflammation and suppresses CD11b $\beta 2$-integrin-dependent signaling. Blood 121, 2084-2094 (2013).

58. Sedlacek, H. H. \& Seiler, F. R. Immunotherapy of neoplastic diseases with neuraminidase: contradictions, new aspects, and revised concepts. Cancer Immunol. Immunother. 5, 153-163 (1978).

59. Jandus, C. et al. Interactions between Siglec-7/9 receptors and ligands influence NK cell-dependent tumor immunosurveillance. J. Clin. Invest. 124, 1810-1820 (2014).

60. Wang, J. et al. Siglec-15 as an immune suppressor and potential target for normalization cancer immunotherapy. Nat. Med. 25, 656-666 (2019).

61. Tsui, C. K. et al. Systematic identification of regulators of antibody-drug conjugate toxicity using CRISPR-Cas9 screens. bioRxiv 557454 (2019). doi:10.1101/557454

62. Gasteiger, E. et al. ExPASy: the proteomics server for in-depth protein knowledge and analysis. Nucleic Acids Res. 31, 3784-3788 (2003).

\section{Acknowledgements}

We thank Drs. Steven Banik, CJ Cambier, and Simon Wisnovsky for their critical reading of this manuscript. We thank Theresa McLaughlin and the Stanford University Mass Spectrometry (SUMS) facility for performing intact protein characterization and HRMS analysis. We are grateful to the Zippelius lab for kindly providing the HER2 ${ }^{+}$ EMT6 cell line, Dr. Eric R. Vimr for the gift of the plasmid pCVD364, Dr. David Rabuka (Catalent Pharma Solutions) for kindly providing humanized trastuzumab with an aldehyde tag and Dr. Mason Appel for generating the pET28-MBP-tev-tb-FGE plasmid used to make SMARTag antibodies. We thank Dr. Søren Christensen for the AU54pET9d* plasmid and Dr. Jennifer Kohler for the pGEX-Neu2 construct.

\section{Funding}

This work was supported in part by the Goldschmidt-Jacobson Foundation (to H.L), the Promedica Foundation (to M.A.S) and a Swiss National Science Foundation grant (SNSF Nr. 310030_184720/1), as well as a grant from the National Institutes of Health to C.R.B (NIH CA227942). M.A.G. was supported by the National Science Foundation Graduate Research Fellowship (NSF GRFP) and the Stanford ChEM-H Chemistry/Biology Interface Predoctoral Training Program. N.R.M. was supported by 
the Swiss Government Excellence Scholarship for Foreign Scholars and Artists (FCS) and the Conselho Nacional de Desenvolvimento Científico e Tecnológico. S.A.M. was supported by an NIH F32 Postdoctoral Fellowship. J.T.T. received support from the Stanford Undergraduate Summer Research Program in Chemistry (Funding: Stanford VPUE/UAR). G.A. was supported by the NSF GRFP. P.A.W. was supported by the NSF GRFP and the Stanford ChEM-H Program. E.C.W. was supported by US National Institutes of Health Predoctoral Fellowship F31CA200544.

\title{
Author contributions
}

M.A.G., H.X., E.C.W, and C.R.B. conceived the project. M.A.G., M.S., H.X., J.F.A.P., N.R.M., S.A.M., J.T., C.L.M. G.A., and P.A.W., carried out experiments and interpreted data. M.A.G and C.R.B wrote the manuscript with input from all authors. H.L. and C.R.B. provided supervision.

\section{Competing interests}

M.A.G, H.X., E.C.W., and C.R.B., are inventors of the patent filed by Stanford University (international publication number WO2018006034A1) titled "Conjugates for targeted cell-surface editing" published on January 4, 2018 and licensed by Palleon Pharmaceuticals on 06/27/2017. C.R.B. is a co-founder and Scientific Advisory Board member of Palleon Pharmaceuticals, Enable Bioscience, Redwood Biosciences (a subsidiary of Catalent) and InterVenn Biosciences, and a member of the Board of Directors of Eli Lilly \& Company. H.L. received research and traveling support from Bristol-Myers Squibb. H.L. received traveling support from Merck Sharp Dome and Roche. H.L. is a member of the Scientific Advisory Board of Palleon Pharmaceuticals.

\section{Data and materials availability}

All data are available in the main text or the supplementary materials.

\author{
List of Supplementary materials \\ Supplementary Figures 1-24 \\ Supplementary Methods \\ NMR Spectra \\ Supplementary References
}

